\title{
Effect of donor substituents on thermally activated delayed
}

\section{fluorescence of diphenylsulfone derivatives}

Oleksandr Bezvikonnyi, Dalius Gudeika, Dmytro Volyniuk, Viktorija Mimaite, Bernard Ronit Sebastine, Juozas V. Grazulevicius

\section{Graphical abstract}
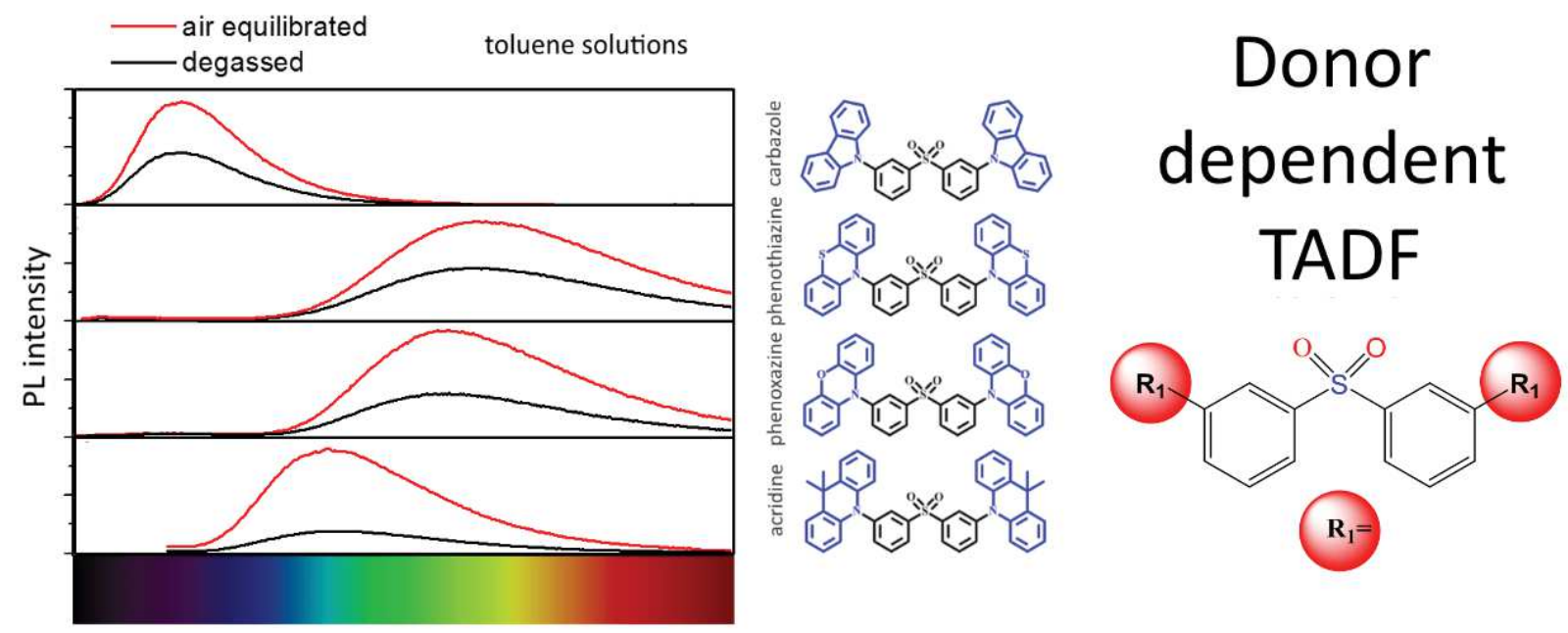


\title{
Effect of donor substituents on thermally activated delayed fluorescence of diphenylsulfone derivatives
}

\author{
Oleksandr Bezvikonnyi, ${ }^{\text {a,b }}$ Dalius Gudeika, ${ }^{\text {a,c }}$ Dmytro Volyniuk, ${ }^{\text {a }}$ Viktorija Mimaite, ${ }^{\text {a Bernard }}$ \\ Ronit Sebastine a and Juozas V. Grazulevicius ${ }^{a^{*}}$
}

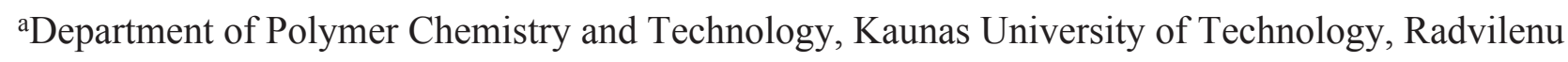
pl. 19, LT-50254, Kaunas, Lithuania

${ }^{b}$ Experimental Physics Department, Faculty of Physics, Taras Shevchenko National University of Kyiv, pros. Akademika Glushkova 4b, Kyiv, Ukraine

${ }^{\mathrm{c}}$ Institute of Solid State Physics, University of Latvia, 8 Kengaraga St., Riga LV-1063, Latvia

* Corresponding author: juozas.grazulevicius@ktu.lt

\begin{abstract}
Diphenylsulfones substituted by acridine, carbazole, phenothiazine and phenoxazine moieties were synthesized and characterized by thermal analysis, UV-, steady-state and time-resolved luminescent spectrometries, cyclic voltametry. Quantum chemical calculations on the molecular level were performed to interpret photophysical properties of the derivatives. Structural parameters, electronic properties, HOMO-LUMO gaps, molecular orbital densities, ionization potentials, reorganization energies were determined. The lowest excitation energies and the wavelengths of absorption maxima were also estimated using the time-dependent density functional theory. All the compounds were found to be capabale to form glasses with glass transition temperatures ranging from 82 to $91{ }^{\circ} \mathrm{C}$. They exhibited high thermal stabilities, with $5 \%$ weight loss temperatures exceeding $385{ }^{\circ} \mathrm{C}$. Strong solvatochromism arising from the intramolecular charge transfer in the excited state was evidenced by bathochromic shifts of
\end{abstract}


emission maxima with increasing solvent polarity. The compounds containing acridine and phenoxazine moieties showed relatively high photoluminescence quantum yield (up to $35 \%$ ) in the non-doped solid state, long delayed fluorescence lifetime (in $\mu$ s range) and small singlet-triplet energy splitting $\left(\Delta \mathrm{E}_{\mathrm{ST}}\right)$ that is attributed to thermally activated delayed fluorescence. These compounds were tested as emissive species for the fabrication of OLEDs. The sky-blue and green devices showed maximum brightness of 3200 and $12300 \mathrm{~cd} / \mathrm{m}^{2}$ and maximum external quantum efficiency of 6.3 and $6.9 \%$, respectively.

Keywords: OLED; TADF; diphenylsulphone; emitter.

\section{Introduction}

Thermally activated delayed fluorescence $(\mathrm{TADF})^{1-3}$ is shown by organic molecules due to the reverse intersystem crossing (RISC) 4 from triplet excited to singlet excited states caused by the thermal motion of atoms and the following charge transfer emission (see Figure 1). This approach allows to employ triplet excitons by converting them to singlet excitons for luminescence and overcome spin statistical $25 \%$ limit of internal quantum efficiency of organic light emitting diodes (OLEDs). Employment of TADF emitters for the fabrication of OLEDs became widespread in recent years due to the possibility of replacing high-cost phosphorescent emitters based on rare metals. TADF emission of the solutions in non-polar solvents corresponds to $\pi^{*} \rightarrow \pi$ transition while emission of the solutions in polar solvents corresponds to strong intramolecular charge transfer. ${ }^{5}$ More polar solvent, the bigger bathochromic shift of the emission peaks is observed. To prevent back transfer of excited energy in non-radiative ways in emitting layers it is reasonable to 
dope a TADF compound into a suitable host. ${ }^{6}$ In the host-guest systems locally excited triplet states can be in a resonance with charge transfer state which leads to stronger RISC. ${ }^{7}$

Careful consideration of the interruption of conjugation between donor and acceptor moieties of p-conjugation length and of redox potential are very important for increasing RISC rate constant. In 2012 Adachi et $\mathrm{al}^{8}$ reported on the first class of deep-blue TADF emitters designed using diphenylsulfone moiety as an acceptor. The advantage of this type of acceptor moiety is in tetrahedral geometry of sulfonyl group which helps to avoid conjugation in the compound thus leading to strong intramolecular charge transfer. In addition, diphenylsulfone moiety has electronwithdrawing properties caused by significant electronegativity of oxygen atoms. ${ }^{9}$

Motivated by the above given information, we synthesized and characterized a series of new donor-acceptor-donor type diphenylsulfone-based TADF materials and studied them in host-guest systems in order to disclose relationship between structure and properties and to test the optimized systems in OLEDs. In contract to earlier reported para-donor-substituted diphenylsulfones ${ }^{8}$, in the synthesized compounds donor moieties, i.e. acridine, carbazole, phenothiazine and phenoxazine, are attached to meta positions of diphenylsulfone core.

\section{Material and Methods}

9H-Carbazole, 10H-phenothiazine, 10H-phenoxazine, 9,9-dimethyl-9,10-dihydroacridine, tri-tert-butylphosphine $\left(\mathrm{P}(t-\mathrm{Bu})_{3}\right)$ solution $\left(1.0 \mathrm{M}\right.$ in toluene), palladium(II) acetate $\left(\mathrm{Pd}(\mathrm{OAc})_{2}\right)$, sodium tert-butoxide $(t$-BuONa) were purchased from Aldrich and used as received. Solvents were dried and purified by distillation. Di(3-bromophenyl)sulfone (I) m.p. 97-99 ${ }^{\circ} \mathrm{C}$ was synthesized according to the reported procedure ${ }^{10}$. 


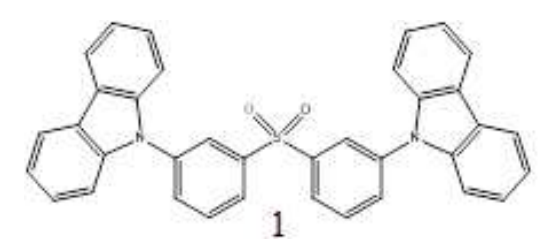

To a solution of di(3-bromophenyl)sulfone (I) (0.5 g, $1.33 \mathrm{mmol}), 18$-crown-6 (0.018 g, $0.066 \mathrm{mmol})$ and $9 H$-carbazole $(0.54 \mathrm{~g}, 3.22 \mathrm{mmol})$ in tetrahydro-1,3-dimethyl-2(1H)pyrimidine (DMPU) $(5 \mathrm{~mL})$ was added $\mathrm{K}_{2} \mathrm{CO}_{3}(0.37 \mathrm{~g}, 2.66 \mathrm{mmol})$. The reaction mixture was purged with nitrogen before adding $\mathrm{CuI}(0.013 \mathrm{~g}, 0.066 \mathrm{mmol})$. The mixture was stirred at $170{ }^{\circ} \mathrm{C}$ for $24 \mathrm{~h}$ under nitrogen, the resulting mixture was cooled to room temperature and poured into water, extracted with chloroform. The organic phase was washed with aqueous $\mathrm{NaCl}$ solution, and dried with anhydrous $\mathrm{Na}_{2} \mathrm{SO}_{4}$. After removal of the solvent by rotary evaporation, the residue was purified by silica gel column chromatography using $n$-hexane/ethylacetate (8:1) to afford $\mathbf{1}$ as a white solid. It was recrystallized from the eluent mixture of solvents. $\mathrm{FW}=548.66 \mathrm{~g} / \mathrm{mol}$; Yield: $0.49 \mathrm{~g}, 67 \%$; m.p. $165-166{ }^{\circ} \mathrm{C} .{ }^{1} \mathrm{H}$ NMR $\left(300 \mathrm{MHz}, \mathrm{CDCl}_{3}, \delta\right.$, ppm): 8.27 (d, $\left.J=8.6 \mathrm{~Hz}, 4 \mathrm{H}\right), 8.09$ - 7.95 (m, 5H), $7.82(\mathrm{~d}, J=8.6 \mathrm{~Hz}, 4 \mathrm{H}), 7.43(\mathrm{~d}, J=7.8 \mathrm{~Hz}, 2 \mathrm{H}), 7.37-7.26(\mathrm{~m}, 5 \mathrm{H}), 6.93\left(\mathrm{dd}, J_{1}=7.8 \mathrm{~Hz}\right.$, $\left.J_{2}=2.2 \mathrm{~Hz}, 4 \mathrm{H}\right) .{ }^{13} \mathrm{C} \mathrm{NMR}\left(75 \mathrm{MHz}, \mathrm{CDCl}_{3}, \delta, \mathrm{ppm}\right): 159.3,142.7,141.2,140.0,139.3,130.0$, $129.7,129.2,127.1,126.5124 .9,124.3124 .1,121.3,121.0,120.8119 .7,117.7,114.7,109.3$, 109.0, 94.3, 94.1, 93.4. $\mathrm{MS}\left(\mathrm{APCI}^{+}, 20 \mathrm{~V}\right), \mathrm{m} / \mathrm{z}: 550\left([\mathrm{M}+\mathrm{H}]^{+}\right)$. Elemental analysis calcd (\%) for $\mathrm{C}_{36} \mathrm{H}_{24} \mathrm{~N}_{2} \mathrm{O}_{2} \mathrm{~S}: \mathrm{C}, 78.81 ; \mathrm{H}, 4.41 ; \mathrm{N}, 5.11 ; \mathrm{O}, 5.83 ; \mathrm{S}, 5.84$; Found: C, 78.78; H, 4.40; N, 5.16.

\subsection{Bis(3-(phenothiazine-10-yl)phenyl) sulfone (2)}




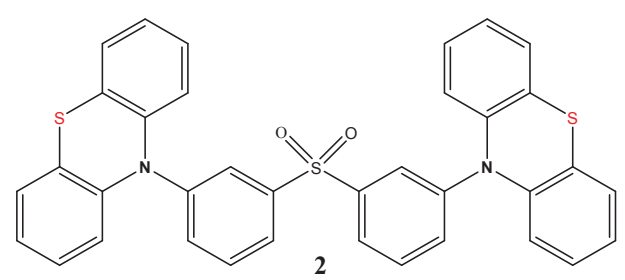

Di(3-bromophenyl)sulfone (I) (0.5 g, $1.33 \mathrm{mmol}), 10 H$-phenothiazine (0.58 g, $2.93 \mathrm{mmol})$, $\mathrm{Pd}(\mathrm{OAc})_{2}(0.012 \mathrm{~g}, 0.049 \mathrm{mmol})$, tri-tert-butylphosphine solution (1.0 M in toluene $)(0.022 \mathrm{~g}, 0.106$ mmol), sodium tert-butoxide $(0.51 \mathrm{~g}, 0.005 \mathrm{mmol})$ and $15 \mathrm{ml}$ of anhydrous toluene were heated at $110^{\circ} \mathrm{C}$ for $24 \mathrm{~h}$ under nitrogen. After cooling, the reaction mixture was diluted with chloroform and the organic phase was washed with water. After being dried over $\mathrm{Na}_{2} \mathrm{SO}_{4}$ and filtered, the solvent was removed and the residue was purified by column chromatography using $n$-hexane/ethylacetate (7:1) as an eluent and crystallized from the eluent mixture of solvents to obtain 2 as yellow crystals. $\mathrm{FW}=612.78 \mathrm{~g} / \mathrm{mol}$; yield 0.48 g, 59\%); m.p. $194-195{ }^{\circ} \mathrm{C} .{ }^{1} \mathrm{H}$ NMR $\left(300 \mathrm{MHz}, \mathrm{CDCl}_{3}, \delta, \mathrm{ppm}\right)$ : $7.85\left(\mathrm{dd}, J_{I}=7.6 \mathrm{~Hz}, J_{2}=1.0 \mathrm{~Hz}, 4 \mathrm{H}\right), 7.62\left(\mathrm{dd}, J_{1}=14.1 \mathrm{~Hz}, J_{2}=5.8 \mathrm{~Hz}, 2 \mathrm{H}\right), 7.48\left(\mathrm{dd}, J_{I}=14.1\right.$ $\left.\mathrm{Hz}, J_{2}=5.8 \mathrm{~Hz}, 2 \mathrm{H}\right), 7.24-7.16(\mathrm{~m}, 4 \mathrm{H}), 7.03-6.95(\mathrm{~m}, 8 \mathrm{H}), 6.53-6.47(\mathrm{~m}, 4 \mathrm{H}) .{ }^{13} \mathrm{C}$ NMR $(75$ $\left.\mathrm{MHz}, \mathrm{CDCl}_{3}, \delta, \mathrm{ppm}\right): 143.9,143.4,142.7,131.2,130.5,127.7,127.1,125.6,124.5,124.3,119.8$. MS (APCI $\left.{ }^{+}, 20 \mathrm{~V}\right), \mathrm{m} / \mathrm{z}: 614\left([\mathrm{M}+\mathrm{H}]^{+}\right)$. Elemental analysis calcd (\%) for $\mathrm{C}_{36} \mathrm{H}_{24} \mathrm{~N}_{2} \mathrm{O}_{2} \mathrm{~S}_{3}: \mathrm{C}, 70.56$; H, 3.95; N, 4.57; O, 5.22; S, 15.70; Found: C, 70.51; H, 3.99; N, 4.61.

\subsection{Bis(3-(phenoxazine-10-yl)phenyl)sulfone (3)}

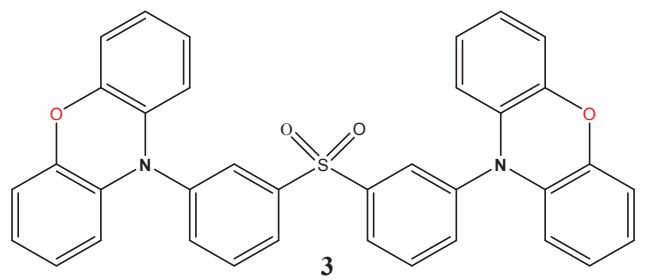

A procedure similar to that used for 2 was followed but with $10 H$-phenoxazine $(0.54 \mathrm{~g}, 2.93$ mmol) instead of $10 H$-phenothiazine. The residue was purified by column chromatography using $n$ - 
hexane/ethylacetate (9:1) as an eluent and crystallized from the eluent mixture of solvents to obtain

3 as yellow crystals. $\mathrm{FW}=580.66 \mathrm{~g} / \mathrm{mol}$; Yield: 0.32 g, 42\%; m.p. 199-200 ${ }^{\circ} \mathrm{C} .{ }^{1} \mathrm{H}$ NMR $(300 \mathrm{MHz}$, $\left.\mathrm{CDCl}_{3}, \delta, \mathrm{ppm}\right): 8.09\left(\mathrm{dd}, J_{1}=7.9 \mathrm{~Hz}, J_{2}=2.9 \mathrm{~Hz}, 2 \mathrm{H}\right), 8.00\left(\mathrm{dd}, J_{1}=5.5 \mathrm{~Hz}, J_{2}=2.9 \mathrm{~Hz}, 2 \mathrm{H}\right)$, $7.82\left(\mathrm{dd}, J_{1}=7.9 \mathrm{~Hz}, J_{2}=5.5 \mathrm{~Hz}, 2 \mathrm{H}\right), 7.65\left(\mathrm{dd}, J_{1}=7.9 \mathrm{~Hz}, J_{2}=2.9 \mathrm{~Hz}, 2 \mathrm{H}\right), 6.76-6.68(\mathrm{~m}, 8 \mathrm{H})$, $6.58-6.48(\mathrm{~m}, 4 \mathrm{H}), 5.78\left(\mathrm{dd}, J_{1}=7.9 \mathrm{~Hz}, J_{2}=2.9 \mathrm{~Hz}, 4 \mathrm{H}\right) .{ }^{13} \mathrm{C} \mathrm{NMR}\left(75 \mathrm{MHz}, \mathrm{CDCl}_{3}, \delta, \mathrm{ppm}\right)$ : $144.2,143.9,140.5,136.6,133.4,132.4,130.6,127.5,123.3,122.1,115.9,113.0,112.8 . \mathrm{MS}$ $\left(\mathrm{APCI}^{+}, 20 \mathrm{~V}\right), \mathrm{m} / \mathrm{z}: 582\left([\mathrm{M}+\mathrm{H}]^{+}\right)$. Elemental analysis calcd (\%) for $\mathrm{C}_{36} \mathrm{H}_{24} \mathrm{~N}_{2} \mathrm{O}_{4} \mathrm{~S}: \mathrm{C}, 74.47 ; \mathrm{H}$, 4.17; N, 4.82; O, 11.02; S, 5.52; Found: C, 74.51; H, 4.11; N, 4.86.

\subsection{Bis(3-(9,9-dimethyl-9,10-dihydroacridine-10-yl)phenyl)sulfone (4)}

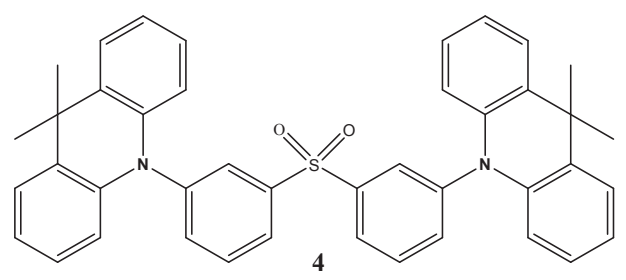

A procedure similar to that used for $\mathbf{2}$ was followed but with 9,9-dimethyl-9,10dihydroacridine $(0.61 \mathrm{~g}, 2.93 \mathrm{mmol})$ instead of $10 \mathrm{H}$-phenothiazine. The residue was purified by column chromatography using $n$-hexane/ethylacetate (8:1) as an eluent and crystallized from the eluent mixture of solvents to obtain 4 as white crystals. FW $=632.82 \mathrm{~g} / \mathrm{mol}$; Yield: $0.51 \mathrm{~g}, 61 \%$; m.p. $259-260{ }^{\circ} \mathrm{C} .{ }^{1} \mathrm{H}$ NMR (300 MHz, $\mathrm{CDCl}_{3}, \delta$, ppm): 8.04 (d, J=8.2 Hz, 2H), 7.99 (t, J=2.2 Hz, 2H), $7.76(\mathrm{t}, J=8.2 \mathrm{~Hz}, 2 \mathrm{H}), 7.59(\mathrm{~d}, J=8.2 \mathrm{~Hz}, 2 \mathrm{H}), 7.48$ (d, $J=2.2 \mathrm{~Hz}, 4 \mathrm{H}), 6.91$ (dd, $J_{1}=8.2$ $\left.\mathrm{Hz}, J_{2}=2.2 \mathrm{~Hz}, 6 \mathrm{H}\right), 6.02(\mathrm{~d}, J=8.2 \mathrm{~Hz}, 6 \mathrm{H}), 1.71(\mathrm{~s}, 12 \mathrm{H}) .{ }^{13} \mathrm{C} \mathrm{NMR}\left(75 \mathrm{MHz}, \mathrm{CDCl}_{3}, \delta, \mathrm{ppm}\right)$ : 144.0, 143.6, 143.0, 138.1, 136.7, 131.9, 130.8, 130.0, 127.0, 123.2, 122.3, 113.3, 112.5, 31.4, 31.2. MS (APCI $\left.{ }^{+}, 20 \mathrm{~V}\right), \mathrm{m} / \mathrm{z}: 634\left([\mathrm{M}+\mathrm{H}]^{+}\right)$. Elemental analysis calcd (\%) for $\mathrm{C}_{42} \mathrm{H}_{36} \mathrm{~N}_{2} \mathrm{O}_{2} \mathrm{~S}: \mathrm{C}, 79.72$; H, 5.73; N, 4.43; O, 5.06; S, 5.07; Found: C, 79.68; H, 5.77; N, 4.41. 


\section{Experimental}

${ }^{1} \mathrm{H}$ and ${ }^{13} \mathrm{C}$ NMR spectra were recorded on a Varian Unity Inova 300 apparatus at ambient temperature. Hydrogen nuclei $\left({ }^{1} \mathrm{H}\right)$ were excited using the frequency of $300 \mathrm{MHz}$, and carbon nuclei $\left({ }^{13} \mathrm{C}\right)$ were excited with the $75 \mathrm{MHz}$ frequency. Spectrum scale is divided into parts per million $(\mathrm{ppm})$. The presented chemical shifts $(\delta)$ are relative to with tetramethylsilane as the internal standard. IR spectra were recorded on a Perkin-Elmer Spectrum GX II FT-IR System spectrometer. Mass spectra were recorded either on a Waters ZQ 2000 analytical system. Elemental analysis was performed with an Exeter Analytical CE-440 Elemental Analyzer. Melting points were recorded on Electrothermal MEL-TEMP melting point apparatus. Differential scanning calorimetry (DSC) measurements were carried out using a DSC Q2000 calorimeter (TA Instruments) under nitrogen environment. Thermogravimetric analysis (TGA) was performed on a TA Instruments Q50 apparatus under nitrogen gas. Cyclic voltammetry measurements were performed on a $\mu$-Autolab Type III potentiostat (EcoChemie, Netherlands). Electrochemical experiments were carried out at room temperature using a three-electrode cell consisting of a platinum coil as counter electrode, a glassy carbon working electrode, and a silver wire as reference electrode. $0.1 \mathrm{M}$ solution of tetrabutylammonium hexafluorophosphate $\left(n-\mathrm{Bu}_{4} \mathrm{NPF}_{6}\right)$ was used as supporting electrolyte at a scan rate of $100 \mathrm{mVs}^{-1}$. For the measurements, silver reference electrode was calibrated against ferrocene/ferrocenium $\left(\mathrm{Fc} / \mathrm{Fc}^{+}\right)$redox couple as an internal standard ${ }^{11}$.

Edinburgh Instruments FLS980 and Aventes AvaSpec-2048XL spectrometers were used for recording photoluminescence. The photoluminescence quantum yields of samples were taken at room temperature by an absolute method using the FLS980 integrating sphere excited at $330 \mathrm{~nm}$ with the Xe lamp. FLS980 and a PicoQuant LDH-D-C-375 laser (wavelength $374 \mathrm{~nm}$ ) as the excitation source were used to measure PL decay curves and PL intensity dependencies on laser 
flux of samples at room and low temperatures. Variable temperature liquid nitrogen cryostat (Optistat DN2) was used for photophysical investigations at different temperatures and $\mathrm{N}_{2}$ atmosphere. Preparing of the degassing of the dilute tetrahydrofuran solutions was made by at least 5 freeze/thaw cycles and vacuum pumping. Electroluminescence, optical density spectra were recorded on an Aventes AvaSpec-2048XL spectrometer. OLEDs were fabricated by step-by-step vacuum-deposition of organic and metal layers onto precleaned patterned ITO-coated glass substrates with a sheet resistance of $30 \Omega / \mathrm{sq}$ under the vacuum of $2 \times 10^{-6} \mathrm{mBar}$ using vacuum equipment from Kurt J. Lesker in-built in an MB EcoVap4G glove box. Thermal deposition rates of organic layers were $\sim 1-2 \AA /$ s. Density-voltage and luminanace-voltage characterization were made simultaneously by using Keithley 6517B electrometer, calibrated PH100-Si-HA-D0 photodiode, Keithley 2400C sourcemeter and a PC-Based Power and Energy Monitor 11S-LINK. All electrophysical measurements were done in the air at $293 \mathrm{~K}$ without passivation. The studied materials in solid-state were tested by photoelectron emission spectrometry in air ${ }^{12}$.

For photoelectron emission measurements, the layers of studied compounds were thermally deposited on glass substrates coated by fluorine doped tin oxide (FTO) at vacuum higher than $3 \times 10^{-}$ ${ }^{6}$ mbar utilizing equipment from Kurt J. Lesker in-built in an MB EcoVap4G glove box. To get ionization potentials of the materials in solid-state, photoelectron emission spectra for them were recorded. In the photoelectron emission setup which was described elsewhere ${ }^{13}$, a deep UV deuterium light source ASBN-D130-CM, a CM110 1/8m monochromator, and an electrometer 6517B Keithley were utilized. The vacuum deposited layers of studied materials were characterazed by the time-of-flight (TOF) technique and the carrier extraction in linearly increasing voltage (CELIV) method in the dark- CELIV and photo-CELIV regimes ${ }^{14-15}$. For studied compounds, usage of these methods allowed to obtained data for proving their semiconducting properties, for determining of type of conductivity and for calculating charge carrier mobility at certain electric 
fields. In the TOF measurements, the transit times $t_{\text {tr }}$ for the samples were found for calculating charge carrier mobility by the formula $\mu=d^{2} U^{-1} t_{\mathrm{tr}}{ }^{-1}$, where $d$ is the layer thickness, and $U$ is the surface potential at the moment of illumination. In the CELIV experiments, the extraction times $\left(t_{\max }\right)$ at the maxima of CELIV photocurrent transients were obtained for calculating charge carrier mobility by the equation $\mu=2 d^{2} A^{-1} t^{-2}$ max ; where $A=U(t) / t$ is the voltage rise rate, $U(t)$ and $t$ are amplitude and triangle pulse duration, respectively ${ }^{14}$. In setup of the both techniques, the same equipment was involved such as the pulsed Nd:YAG laser (EKSPLA NL300, a wavelength of 355 nm, pulse duration 3-6 ns), the Keithley 6517B electrometer, the Tektronix TDS 3052C oscilloscope and the function generator Tektronix AFG3011C. Density function theory (DFT) employing B3LYP/6-31G(d,p) were performed with the Gaussian 16. The molecular orbitals of the molecules were visualized using Gaussview 6. The spectroscopic properties of the compounds were investigated by means of the time dependent DFT (TD-DFT) with B3LYP/6-31G(d,p). Up to 40 excited states were used for the estimation of the theoretical absorption spectra. A natural transition orbitals (NTO) were used to visualize electron transitions in compound $\mathbf{1}$ at triplet excited state. The vertical ionization potentials (IP) were calculated at the B3LYP/6-31G(d,p) level as energy difference between neutral and cation radical species at the neutral state geometry.

\section{Results and Discussion}

\section{Synthesis and characterization}

Synthetic routes to the target compounds are shown in Scheme 1. Procedures used to prepare derivatives 1-4 are presented in the Experimental section (Supporting Information). 
The key intermediate di(3-bromophenyl)sulfone (I) was synthesized via bromination by $\mathrm{N}$ bromosuccinimide in sulfonic acid with diphenyl sulfone as the starting material. Ullmann coupling reaction of bromo-substituted diphenyl sulfone (I) with $10 H$-carbazole led to compound $\mathbf{1}$. The target derivatives 2, 3 and $\mathbf{4}$ were synthesized by Pd-catalyzed Buchwald-Hartwig cross-coupling reactions between di(3-bromophenyl)sulfone (I) and 10H-phenothiazine, 10H-phenoxazine or 9,9dimethyl-9,10-dihydroacridine. The products were purified by the silica column chromatography and recrystallized from the eluent mixtures of solvents yielding pure crystals. ${ }^{1} \mathrm{H}$ NMR, ${ }^{13} \mathrm{C}$ NMR, mass spectrometries, and elemental analysis were employed to confirm the chemical structures of 14. The compounds were soluble in common organic solvents such us dichloromethane, tetrahydrofuran (THF), acetone, toluene etc.

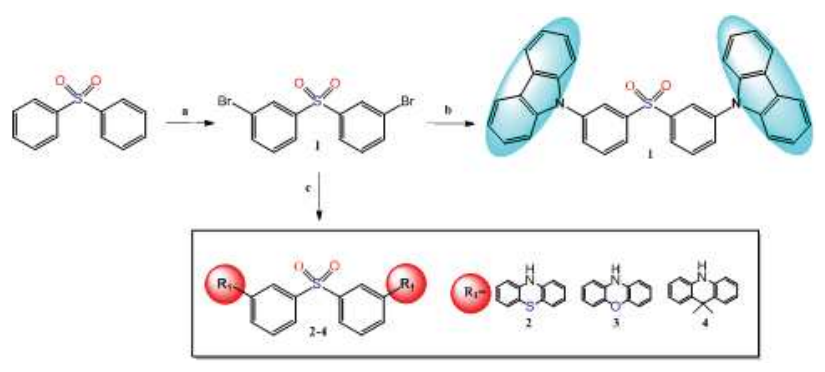

Scheme 1. Synthetic routes to 1-4. Reagents and conditions: (a) N-bromosuccinimide, sulfonic acid (conc.), $70{ }^{\circ} \mathrm{C}, 2 \mathrm{~h}$; (b) $9 \mathrm{H}$-carbazole, 18-crown-6, CuI, DMPU, $\mathrm{K}_{2} \mathrm{CO}_{3}, 170$ ${ }^{\circ} \mathrm{C}, 24 \mathrm{~h}$; (c) 10H-phenothiazine, 10H-phenoxazine or 9,9-dimethyl-9,10-dihydroacridine, $\mathrm{Pd}(\mathrm{OAc})_{2}, \mathrm{P}(t-\mathrm{Bu})_{3}, t$-BuONa, toluene, $110^{\circ} \mathrm{C}, 24 \mathrm{~h}$.

\section{Geometry and frontier orbitals}

For insight into the impact of different donor moieties attached to the diphenylsulfone on properties of compounds 1-4, quantum chemical calculations were performed. DFT calculations were carried out using B3LYP/6-31g(d,p) level to compute geometries of the compounds at ground state $\left(\mathrm{S}_{0}\right)$ (Figure 1). For compound 1 the dihedral angle between carbazole and phenyl plane of the 
diphenylsulfone moiety is only $50^{\circ}$ which does not eliminate the coupling between donor and acceptor fragments. Therefore, HOMO is localized on carbazole and phenyl units with less electron contribution from the second carbazole fragment. For 2-4, corresponding the dihedral angles between donor and acceptor fragments range from 81 to $91^{\circ}$. Due to large dihedral angles, these molecules demonstrate small spatial overlap between HOMO and LUMO. HOMO of 2-4 are localized on acridine, phenothiazine and phenoxazine moieties, while the LUMO of all four compounds is dispersed essentially over electron deficient diphenylsulfone unit.

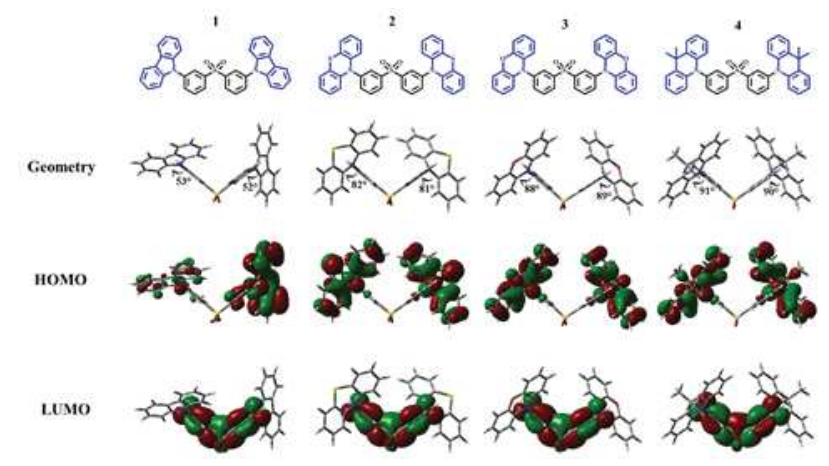

Figure 1. Geometries and frontier orbitals of compounds 1-4 calculated at B3LYP/6-31(d,p) level in the absence of medium effect.

\section{Thermal properties}

Thermal properties of compounds $\mathbf{1 - 4}$ were studied by thermogravimetric analysis (TGA) and differential scanning calorimetry (DSC). The data are summarized in Table 1. TGA curves are shown in Supporting Information (Figure S1). The 5\% weight loss temperatures ( $\mathrm{T}_{\mathrm{ID}-5 \%}$ ) of 1-4 ranged from 339 to $385^{\circ} \mathrm{C}$. Compounds $\mathbf{2 - 4}$ containing phenothiazinyl, phenoxazinyl and acridinyl moieties exhibited higher $\mathrm{T}_{\mathrm{ID}-5 \%}$ (Table 1) compared to compound $\mathbf{1}$ containing carbazolyl moieties. Compound 1 also showed the lowest melting point. These observations can apparently be explained by the weaker intermolecular interaction in the crystals of $\mathbf{1}$.

Table 1. Thermal characteristics of compounds 1-4. 


\begin{tabular}{cccc} 
Compound & $\mathrm{T}_{\mathrm{m}},\left[{ }^{\circ} \mathrm{C}\right]^{[\mathrm{a}]}$ & $\mathrm{T}_{\mathrm{g}},\left[{ }^{\circ} \mathrm{C}\right]^{[\mathrm{b}]}\left(2^{\text {nd }}\right.$ heating scan $)$ & $\mathrm{T}_{\mathrm{ID}}-5 \%,\left[{ }^{\circ} \mathrm{C}\right]^{[\mathrm{c}]}$ \\
\hline $\mathbf{1}$ & 169 & 84 & 339 \\
\hline $\mathbf{2}$ & 198 & 82 & 385 \\
\hline $\mathbf{3}$ & 205 & 85 & 374 \\
\hline $\mathbf{4}$ & 265 & 91 & 363
\end{tabular}

[a] $\mathrm{T}_{\mathrm{m}}$ is melting temperature at the scan rate of $10^{\circ} \mathrm{C} / \mathrm{min}, \mathrm{N}_{2}$ atmosphere. [b] $\mathrm{T}_{\mathrm{g}}$ is glass-transition temperature. [c] $\mathrm{T}_{\mathrm{ID}}{ }^{-5} \%$ is $5 \%$ weight loss temperature at the scan rate of $20^{\circ} \mathrm{C} / \mathrm{min}, \mathrm{N}_{2}$ atmosphere.

Diphenylsulfone-based compounds 1-4 were isolated after the synthesis as crystalline substances. However, they could be transformed into molecular glasses by cooling their melts as it was confirmed by DSC. DSC thermograms of compound $\mathbf{3}$ are shown in Figure 2. When the sample of $\mathbf{3}$ was heated during DSC experiment the endothermal melting signal was observed at $205{ }^{\circ} \mathrm{C}$. The cooling scan did not show any signal of crystallization. The following heating scan revealed only glass transition at $85^{\circ} \mathrm{C}$. The similar behavior was observed for compounds $\mathbf{1}, \mathbf{2}$ and 4. In the first heating scans compounds $\mathbf{1 , 2}$ and $\mathbf{4}$ showed endothermic melting signals at 169, 198, and $265{ }^{\circ} \mathrm{C}$, respectively. In the repeated heating scans these compounds showed only glass transitions at 84,82 and $91^{\circ} \mathrm{C}$, respectively.

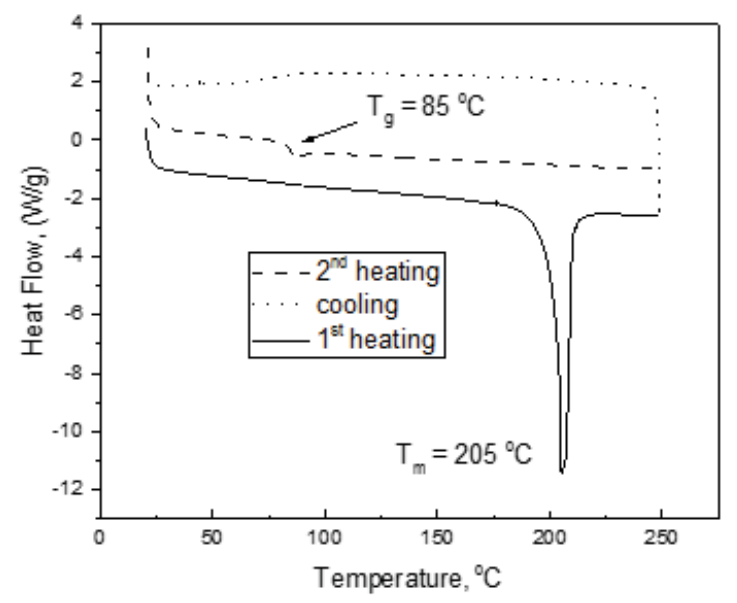

Figure 2. DSC thermograms of compound 3.

\section{Photophysical properties}

Absorption and photoluminescence (PL) spectra of dilute solutions of compounds 1-4 recorded at room temperature are presented in Figure 3a. Photophysical data are collected in 
Tables 2-4. Theoretical and experimental absorption spectra of the compounds are in good agreement (Figure 3a, S2). The theoretical results indicate presence of the several excited sates of charge transfer (CT) nature exhibiting practically zero oscillator strength with negligible conjugation as it was observed for most of TADF compounds. ${ }^{16}$ The lowest energy bands (LEB) of the compounds demonstrate contributions of electronic transitions towards various excited states. Position and intensity of the LEB strongly depends on the type of donor moiety attached. Due to the enhanced intermolecular interactions the films exhibit broader and slightly red-shifted absorption (see Figure 3b). ${ }^{17-18}$ However, as it can be seen from the edge of absorption of 4 (Figure 3a) methyl groups attached to acridine moiety prevent to the great extent the intermolecular interactions in the neat solid sample of the compound. Knowing the absorption onset it is possible to find optical band gaps $\left(E_{g}{ }^{o p t}\right)$ values using correlation $1239.84 / \lambda_{\text {onset. }} E_{g}{ }^{o p t}$ for the solid films of compounds 1-4 were found to be 3.32, 3.23, 3.19 and $3.70 \mathrm{eV}$, respectively. PL spectra (Figure 3a) of 1-4 are broad and red-shifted with respect to the corresponding absorption spectra. Stokes shift of $\mathbf{1}$ is sufficiently small to allow self-quenching by Förster resonance energy transfer (FRET). ${ }^{19-20}$ Two main effects lead to the large Stokes shifts up to $300 \mathrm{~nm}$ observed for the solutions of 2-4 in polar THF: (i) intramolecular charge transfer (ICT) due to almost orthogonal inter-ring geometry between donor and acceptor; (ii) strong electron donating ability of phenothiazine, phenoxazine and acridane moieties. ${ }^{21}$ Replacement of the solvent with the less polar one $\left(\right.$ THF $\left(\varepsilon_{\mathrm{r}}=7.5\right) \rightarrow$ chloroform $\left(\varepsilon_{\mathrm{r}}=4.81\right) \rightarrow$ toluene $\left.\left(\varepsilon_{\mathrm{r}}=2.38\right)\right)$ leads to the hypsochromic shift of the emission peaks which is the evidence of ICT nature.

Considering the potential application of compounds $\mathbf{1 - 4}$ as emitters in OLEDs, photophysical properties of solid films of molecular mixtures of 1-4 with mCP and TCz1 were studied. Due to FRET singlet excitons of a host approach singlet states of an emitter ${ }^{19}$ and due to Dexter energy transfer triplet excitons are transferred into the triplet state of an emitter ${ }^{22}$. 
Absorption and PL spectra of the molecular mixtures recorded at room temperature are shown in Figure 3b. Absorption spectra of the molecular mixtures correspond to the absorption of the hosts while emission peaks and their positions correlate with PL spectra of 1-4 however with some hypsochromic shifts. This observation confirms electronic excitation energy transfer from the host to the guest. However, emission spectra of compounds 1-4 doped into TCz1 clearly reflect slight contribution of the host in the high energy range. We can see also the vibronic bands in case of $\mathbf{1}$ doped in TCz1. The values of the photoluminescence quantum yields (PLQY) (Table 3) and PL lifetimes of doped and neat thin films (Table S1) were measured at room temperature (see Figures S3-S14). Compound 4 was recently reported by $\mathrm{Wu}$ et al. ${ }^{23}$ PLQY of the deoxygenated film of 4 with the best film homogenity was found to be of $76 \%$ which correlates well with our data (Table 3, Figure 4). It is possible to achieve much higher values of PLQY for 1-4 compounds in deoxygenated environment. The host-guest systems exhibit comparable and mostly higher PLQY than neat films. This observation can apparently be explained by decrease of extent of concentration quenching in the presence of excitation energy transfer from host to guest. PL decay curves of the solid samples required double exponential fits. Delayed fluorescence with the lifetime in the $\mu$ s range was recorded for all solid samples of $\mathbf{3}$ and $\mathbf{4}$ and can be attributed to TADF. PL and phosphorescence spectra of dilute THF solutions of compounds recorded at liquid nitrogen temperature are presented in Figure S15. Singlet $\left(\mathrm{E}_{\mathrm{S} 1}\right)$ and triplet $\left(\mathrm{E}_{\mathrm{T} 1}\right)$ energies were estimated from the values of the onsets of PL and phosphorescence spectra respectively. The optimized singlet $\left(\mathrm{S}_{1}\right)$ and triplet $\left(\mathrm{T}_{1}\right)$ excited state geometries and the adiabatic transition energies were calculated using time-dependent DFT (TD-DFT). The data are collected in Table $\mathbf{3}$ and presented in Figure S16. In the case of compound $\mathbf{1}, \mathrm{S}_{1}$ state shows CT from carbazole to diphenylsulfone moieties. In $\mathrm{T}_{1}$ state, HONTO involves donor $\rightarrow$ acceptor $\mathrm{CT}$ transition and additionally contribution from the local acceptor unit, while LUNTO is localized mainly on the acceptor unit. ${ }^{24-25}$ The 
separated $S_{1}$ and overlapped $T_{1}$ leads to the singlet-triplet energy splitting up to $0.44 \mathrm{eV}$ for the dilute THF solution of $\mathbf{1} .^{26}$ Both the excited states of compounds 2-4 correspond exclusively to transition from HOMO to LUMO, with both orbitals being strictly localized on the donor- and acceptor moieties, respectively. The twisted structures of 2-4 are responsible for small singlet-triplet energy splittings from $0.01 \mathrm{eV}$ to $0.06 \mathrm{eV}$.

Figure 4 shows the comparison of PL spectra of degassed toluene solutions (red line) and air equilibrated ones (black line) of compounds 1-4 recorded at room temperature.

Table 2. Photophysical parameters derived from steady-state spectroscopic measurements of dilute solutions and solid films of compounds 1-4 in THF.

\begin{tabular}{|c|c|c|c|c|c|c|c|c|c|c|c|}
\hline & $\begin{array}{r}\text { Dilut } \\
\text { sol }\end{array}$ & $\begin{array}{l}\text { THF } \\
\text { ion }\end{array}$ & $\begin{array}{r}\text { Dilut } \\
\text { so }\end{array}$ & $\begin{array}{l}\text { oluene } \\
\text { ion }\end{array}$ & $\begin{array}{r}\mathrm{D} \\
\text { chlo } \\
\text { sol }\end{array}$ & $\begin{array}{l}\text { ate } \\
\text { form } \\
\text { tion }\end{array}$ & & & Neat film & & \\
\hline : & $\begin{array}{c}\text { Abs. } \\
\text { band, } \\
\text { nm }\end{array}$ & $\begin{array}{c}\text { Em. } \\
\text { band, } \\
\mathrm{nm}\end{array}$ & $\begin{array}{c}\text { Abs. } \\
\text { band, } \\
\mathrm{nm}\end{array}$ & $\begin{array}{c}\text { Em. } \\
\text { band, } \\
\mathrm{nm}\end{array}$ & $\begin{array}{c}\text { Abs. } \\
\text { band, } \\
\text { nm }\end{array}$ & $\begin{array}{c}\text { Em. } \\
\text { band, } \\
\mathrm{nm}\end{array}$ & $\begin{array}{c}\text { Abs. } \\
\text { band, } \\
\text { nm }\end{array}$ & $\begin{array}{c}\text { Em. } \\
\text { band, } \\
\mathrm{nm}\end{array}$ & $\begin{array}{c}\begin{array}{c}\text { Stokes } \\
\text { shift, } \\
\text { nm }\end{array}\end{array}$ & $\begin{array}{c}\text { UV: } \lambda_{\text {ed }} \\
\text { ge, } \\
\text { nm }\end{array}$ & $\begin{array}{c}E_{g}^{o p t} \\
, \\
\mathrm{eV}\end{array}$ \\
\hline 1 & 323 & 426 & 338 & 405 & 338 & 422 & 341 & 409 & 68 & 373 & 3.32 \\
\hline 2 & 317 & 596 & 308 & 547 & 315 & 572 & 322 & 543 & 221 & 384 & 3.23 \\
\hline 3 & 305 & 596 & 304 & 561 & 296 & 579 & 320 & 558 & 240 & 389 & 3.19 \\
\hline 4 & 281 & 522 & 301 & 489 & 287 & 515 & 288 & 506 & 218 & 335 & 3.70 \\
\hline
\end{tabular}

(a) 


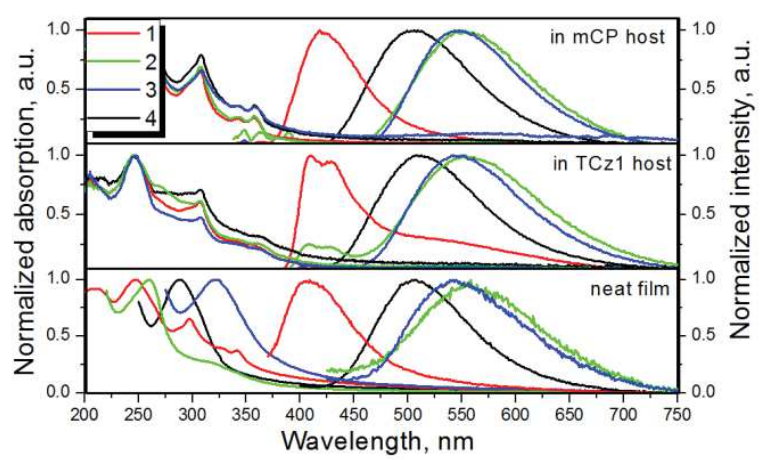

(b)

Figure 3. UV and PL spectra of dilute THF, toluene and chloroform solutions of compounds 1-4 (a) and of thin films of 1-4 doped in $\mathrm{mCP}(5 \mathrm{wt} \%)$ and in $\mathrm{TCz} 1(25 \mathrm{wt} \%)$ as well as of neat thin films(b)

Table 3. Photophysical parameters derived from steady state and time resolved spectroscopic measurements of dilute THF solutions and thin films of compounds 1-4 at 77 $\mathrm{K}$ and at room temperature.

\begin{tabular}{ccccccc} 
Comp. & $\begin{array}{c}\Phi_{\mathrm{PL} \text { TCZ1 }} \\
{[\mathrm{a}], \%}\end{array}$ & $\begin{array}{c}\Phi_{\mathrm{PL} \mathrm{mCP}} \\
{[\mathrm{b}], \%}\end{array}$ & $\begin{array}{c}\Phi_{\mathrm{PL} \text { neat }} \\
{[\mathrm{c}], \%}\end{array}$ & $\mathrm{E}_{\mathrm{S} 1}{ }^{[\mathrm{d}]}, \mathrm{eV}$ & $\mathrm{E}_{\mathrm{T} 1}{ }^{[\mathrm{d}]}, \mathrm{eV}$ & $\Delta \mathrm{E}_{\mathrm{ST}}{ }^{[\mathrm{d}]}, \mathrm{eV}$ \\
\hline $\mathbf{1}$ & 10.9 & 21.8 & 24.4 & $3.49(3.01)$ & $3.05(2.84)$ & $0.44(0.17)$ \\
\hline $\mathbf{2}$ & 8.2 & 11.6 & 9.2 & $2.65(2.24)$ & $2.64(2.22)$ & $0.01(0.02)$ \\
\hline $\mathbf{3}$ & 20.8 & 32.1 & 11.9 & $2.84(2.17)$ & $2.78(2.14)$ & $0.06(0.02)$ \\
\hline $\mathbf{4}$ & 23.9 & 36.5 & 33.1 & $2.97(2.53)$ & $2.92(2.50)$ & $0.05(0.02)$
\end{tabular}

[a] PLQY of the films of compounds doped in TCz1 (25wt\%). [b] PLQY of the films of compounds doped in mCP (5wt\%). [c] PLQY of the neat films. [d] Estimated from the PL spectra of the dilute THF solutions at $77 \mathrm{~K}$. The theoretical values of $\mathrm{E}_{\mathrm{S} 1}, \mathrm{E}_{\mathrm{T} 1}$ and $\Delta \mathrm{E}_{\mathrm{ST}}$ are presented in parentheses.

Upon removing air, the intensity increased by factors of 1.9-4.7 while shapes of the spectra remained unchanged. This observation confirms contribution of the triplet excited states into ${ }^{1} \mathrm{CT}$ emission. ${ }^{16}$ Considering small $\Delta \mathrm{E}_{\mathrm{ST}}$ and the presence of component with the lifetime in a $\mu$ s range 
in PL decay curves of $\mathbf{3}$ and $\mathbf{4}$ it can be presumed that increase of PL intensity after degassing of the solutions is associated with TADF.

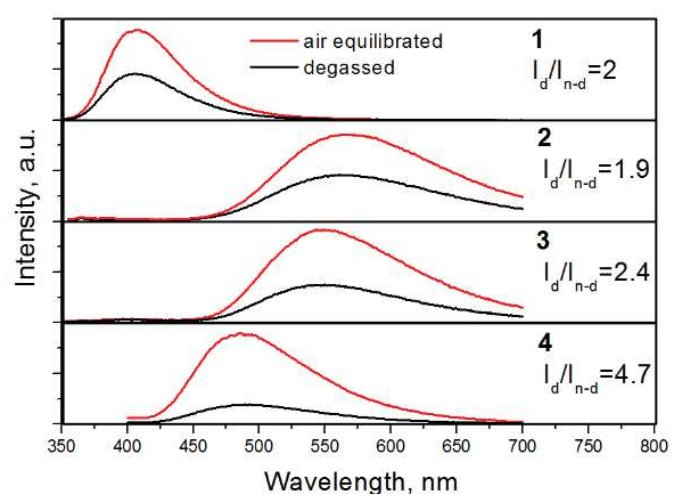

Figure 4. PL spectra of degassed and air equilibrated dilute toluene solutions of 1-4 recorded at room temperature.

Figure 5a shows time resolved emission spectra of $25 \mathrm{wt} \%$ solid solutions of $\mathbf{3}$ and $\mathbf{4}$ in TCz1 recorded at $293 \mathrm{~K}$. Pronounced DF after 474ns delay is observed and the position of the emission peaks remains. This observation shows that radiative way of deactivation for the triplet and singlet excitons is the same. Phosphorescence spectra of the samples recorded at $77 \mathrm{~K}$ are given in Figure 5b. The triplet excited state energy values for the films of the solid solutions of $\mathbf{3}$ and $\mathbf{4}$ in TCz1 were estimated from the phosphorescence spectra and found to be 2.74 and $2.86 \mathrm{eV}$ respectively, while their singlet excited state energy values were found to be 2.75 and $2.90 \mathrm{eV}$, respectively. The values of energy splitting between ${ }^{1} \mathrm{CT}$ and ${ }^{3} \mathrm{CT}\left(\Delta \mathrm{E}_{\mathrm{ST}}\right)$, obtained from the spectra of dilute THF solutions and of solid doped TCz1 films measured at $77 \mathrm{~K}$ were found to be considerably lower than $0.1 \mathrm{eV}$. High polarity of THF is apparently the reason of the slightly bigger $\Delta \mathrm{E}_{\mathrm{ST}}$ values observed for dilute THF solutions relative to those of the doped solid-state films. Thermal activation of the DF in the doped TCz1 films was estimated by the measurements of PL decays at the temperatures from 77 to $300 \mathrm{~K}$ (Figures 6a and S15). With the increase of temperature intensity of long-lived component increases and becomes constant after reaching 
temperatures higher than ca. $260 \mathrm{~K}$, apparently because of small energy barrier between ${ }^{1} \mathrm{CT}$ and ${ }^{3} \mathrm{CT}$ and possible RISC. With the increase of temperature, the shape of PL spectra remains unchanged with the slight red shifts (Figures $\mathbf{6 b}$ and S17). This observation shows that ${ }^{1} \mathrm{CT}$ emission is the only route of radiative deactivation and bathochromic shifts correspond to relaxation of ${ }^{1} \mathrm{CT}$ state, most likely caused by local dipolar interaction between host and guest molecules at higher temperatures. ${ }^{27}$ These results and the linear dependence of the log-log plot of DF intensity (1 $\mu$ s delay) versus excitation laser fluence with the slope of 0.91-0.96 in the entire range of excitation power (Figures $\mathbf{6 b}$ and S18-S20), confirm that pure intramolecular TADF is characteristic of thin films of the molecular mixtures of $\mathbf{4}$ and $\mathbf{3}$ doped with TCz1 $(25 \mathrm{wt} \%)$.

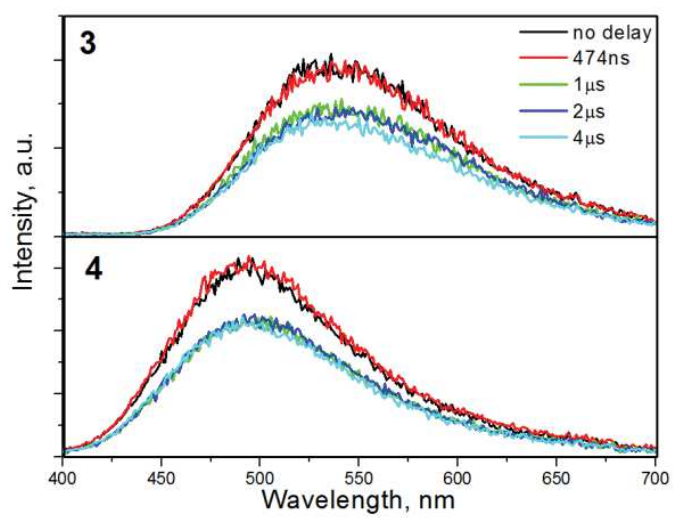

(a)

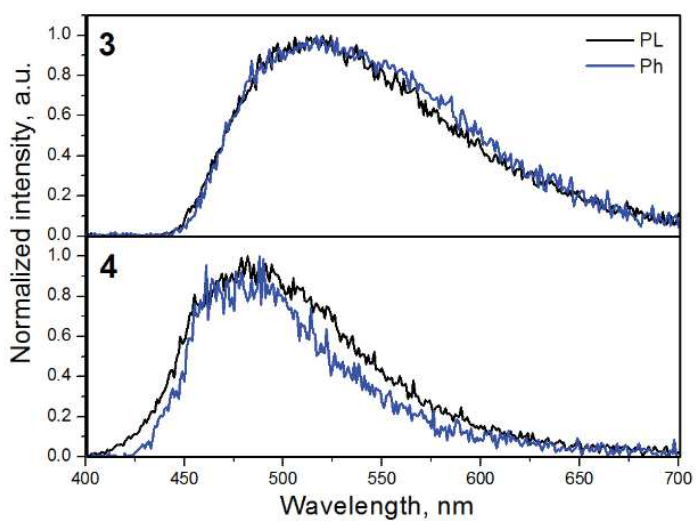

(b) 
Figure 5. Time resolved PL spectra which were recorded with different delays for the films of $25 \mathrm{wt} \%$ solid solutions of $\mathbf{3}$ and $\mathbf{4}$ in TCz1 recorded at room temperature (a) and at liquid nitrogen temperature (b).

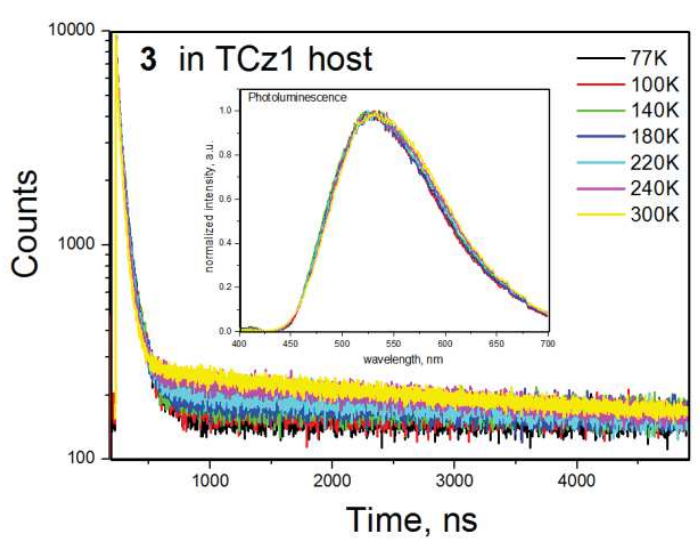

(a)

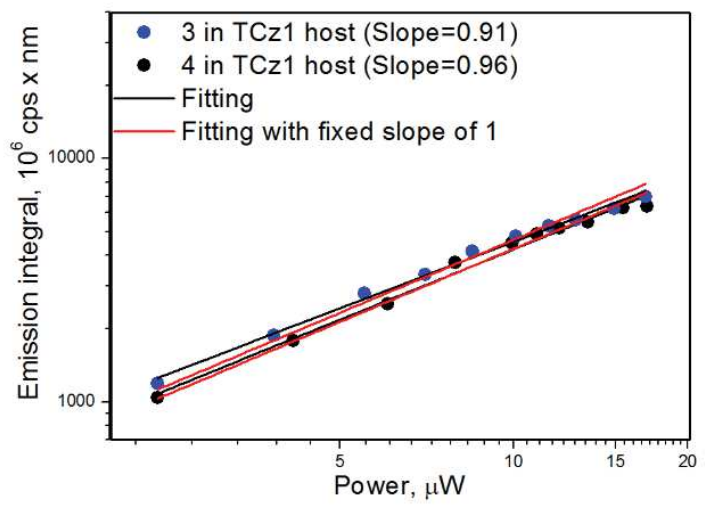

(b)

Figure 6. PL decay curves of the film of 3 doped in TCz1 (25wt\%) at different temperatures. Inset: PL spectra (a). Correlation between DF integral and the excitation dose (b).

Electrochemical and photoelectrical properties

Electrochemical properties of the solutions of 1-4 in dichloromethane were investigated by cyclic voltammetry (CV) using tetrabutylammonium hexafluorophosphate as supporting electrolyte 
and ferrocene as the internal standard. The values of electrochemically established ionization potentials $\left(\mathrm{IP}_{\mathrm{CV}}\right)$ and electron affinity $\left(\mathrm{EA}_{\mathrm{CV}}\right)$ values are presented in Table 4.

Table 4. Electron affinities, ionization potentials and HOMO/LUMO energies of compounds 1-4.

\begin{tabular}{cccccc} 
Compound & $\begin{array}{c}E_{\text {onset }}^{\text {ox }} \\
\mathrm{Fc},[\mathrm{V}]\end{array}$ & $\begin{array}{c}\mathrm{IP}_{\mathrm{CV}^{[\mathrm{a}]}, \mathrm{HOMO}^{\mathrm{c}}}^{[\mathrm{eV}]} \\
\mathbf{1}\end{array}$ & $\begin{array}{c}\mathrm{EA}_{\mathrm{CV}}{ }^{\mathrm{bb}]}, \mathrm{LUMO}^{\mathrm{c}} \\
{[\mathrm{eV}]}\end{array}$ & $\begin{array}{c}\mathrm{IP}, \\
{[\mathrm{eV}]^{[\mathrm{c}]}}\end{array}$ & $\begin{array}{c}\mathrm{IP}_{\mathrm{PE}} \\
{[\mathrm{eV}]^{[\mathrm{d}]}}\end{array}$ \\
\hline $\mathbf{2}$ & 0.86 & $5.80 /-5.60$ & $2.44 /-1.70$ & 6.65 & 5.83 \\
\hline $\mathbf{3}$ & 0.54 & $5.35 /-5.21$ & $1.86 /-1.81$ & 6.26 & 5.53 \\
\hline $\mathbf{4}$ & 0.35 & $5.09 /-4.88$ & $1.72 /-1.83$ & 5.94 & 5.40 \\
\hline & 0.32 & $5.05 /-5.09$ & $1.67 /-1.71$ & 6.09 & 5.51
\end{tabular}

[a] $\mathrm{IP}_{\mathrm{CV}}=\left|-\left(1.4 \times 1 e^{-\times} E_{\text {onset }}^{\text {ox }} \mathrm{vs} \mathrm{Fc} / \mathrm{V}\right)-4.6\right| \mathrm{eV}{ }^{28} ;[\mathrm{b}] \mathrm{EA}_{\mathrm{CV}}=-\left(|\mathrm{IP} \mathrm{CV}|-E_{g}^{\text {opt }}\right)\left(E_{\text {onset }}^{\text {ox }}\right.$ is onset oxidation potential versus the $\mathrm{Fc}^{+} / \mathrm{Fc} ; E_{g}^{o p t}=1240 / \lambda_{\text {edge, }}$ where $\lambda_{\text {edge }}$ is the onset wavelength of absorption spectrum of the dilute chloroform solution in

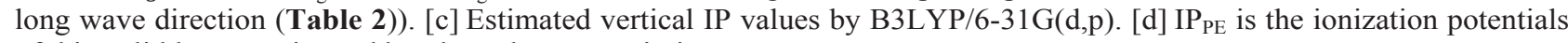
of thin solid layers estimated by photoelectron emission spectrometry.

The shapes of cyclic voltammograms were found to be similar for all the compounds (1-4)

(Figure 7). Compounds 1-4 displayed one oxidation peak, which can be assigned to oxidation of electron-donating carbazole, phenothiazine, phenoxazine and acridane moieties. The first irreversible oxidation of compounds 2-4 took place at $0.54,0.35$ and $0.32 \mathrm{~V}$, respectively, i.e., at lower potentials than for $\mathbf{1}(0.86 \mathrm{~V})$.

According to the method reported by Forrest et al. ${ }^{29}$, ionization energy values $\left(\mathrm{IP}_{\mathrm{CV}}\right)$ values were calculated using the equation $\mathrm{IP}_{\mathrm{CV}}=\left|-\left(1.4 \times 1 \mathrm{e} \times E_{\text {onset }}^{\text {ox }} \mathrm{vs} \mathrm{Fc} / \mathrm{V}\right)-4.6\right| \mathrm{eV}$. The values of $\mathrm{IP}_{\mathrm{CV}}$ are given in Table 4. They ranged from 5.05 to $5.80 \mathrm{eV}$. Compounds $\mathbf{3}$ and $\mathbf{4}$ having phenoxazinyl and acridanyl moieties showed the lowest values of $\mathrm{IP}_{\mathrm{CV}}$. The highest $\mathrm{IP}_{\mathrm{CV}}$ values were observed for carbazolyl and phenothiazinyl-containing compounds $\mathbf{1}$ and 2. Electron affinities $\left(\mathrm{EA}_{\mathrm{CV}}\right)$ 
determined from the optical band gsaps $\left(E_{\text {onset }}^{\text {ox }}\right)$ and ionization energy values were found to range from 1.67 to $2.44 \mathrm{eV}$.

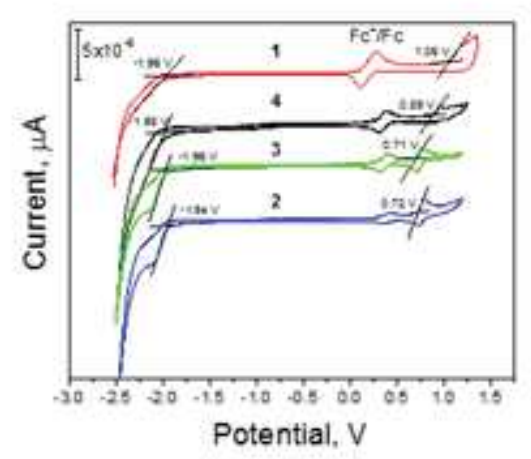

Figure 7. Cyclic voltammograms of dilute solutions of compounds 1-4 in dichloromethane at sweep rcate of $100 \mathrm{mV} / \mathrm{s}$.

Ionization potentials $\left(\mathrm{IP}_{\mathrm{EP}}\right)$ of the solid layers of compounds $\mathbf{1 - 4}$ were estimated by photoelectron emission spectrometry. Photoelectron emission spectra are shown in Figure 8 The values of $\mathrm{IP}_{\mathrm{EP}}$ ranged from 5.40 to $5.80 \mathrm{eV}$. These values were compared with vertical ionization potentials, theoretically calculated in the framework of DFT B3LYP/6-31G(d) approach (Table 4). The trends of the vertical ionization potentials were found to be similar to those estimated by photoelectron emission spectrometry (Figure S21). The lowest values of theoretical vertical IP levels were observed for compounds $\mathbf{3}$ and 4, while the derivatives $\mathbf{1}$ and $\mathbf{2}$ exhibit higher values of IP. It has to be mentioned that the trends of IP are not strictly consistent with the HOMO energy levels. One probable reason is that the B3LYP functional cannot predict orbital energies accurately $^{23}$. 


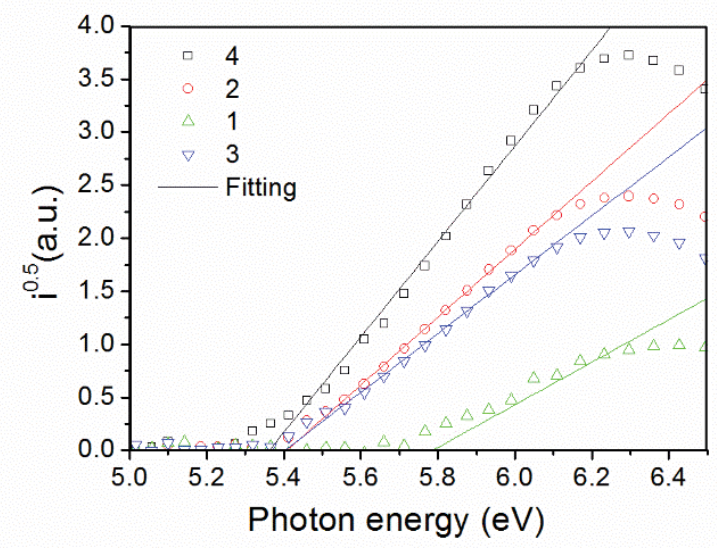

Figure 8. Photoelectron emission spectra of the solid samples of 1-4.

\section{Charge transporting properties}

Using time-of-flight (TOF) technique, ambipolar charge transport for the layers of $\mathbf{2}$ and $\mathbf{3}$ was detected. The transit times for both holes and electrons were well observed in the log-log scales (Figure S22). Experimental TOF mobility values for holes and electrons as a function of applied electric fields for the layers of $\mathbf{2}$ and $\mathbf{3}$ are presented in Figure 9a. The transit times only for electrons were observed for the layer of 4; while, the transit times for both types of charges practically were not possible to detect for the layer of $\mathbf{1}$ because of very dispersive TOF current transients (Figure S22). However, well detectable maxima were observed on photo-CELIV current transients for extracted holes and electrons for the layer of $\mathbf{1}$ (Figure 9b,c). Bipolar charge transport was observed by CELIV for the layers of all the studied diphenylsulfones (Figure S23). Experimental CELIV charge mobility values were in good agreement with those obtained by TOF measurements.

Charge mobility values for the layers of 1-4 ranged from $1 \times 10^{-6}$ to $1 \times 10^{-4} \mathrm{~cm}^{2} / \mathrm{Vs}$ at electric fields ranging from $0.6 \times 10^{5}$ to $8.1 \times 10^{5} \mathrm{~V} / \mathrm{cm}$. These charge mobility values are not very high as for low-molar-mass organic semiconductors. However, it should be noted that these emissive organic semiconductors were deliberately designed to have big angle between donor and acceptor units for 
getting TADF, which obviously does not lead to the enhancement of charge mobility. Electron mobilities were found to be higher than hole mobilities for all the compounds, indicating strong accepting properties of diphenylsulfone unit. Nevertheless, these compounds in tandem with appropriated hosts can be regarded as promising emitters for OLEDs.

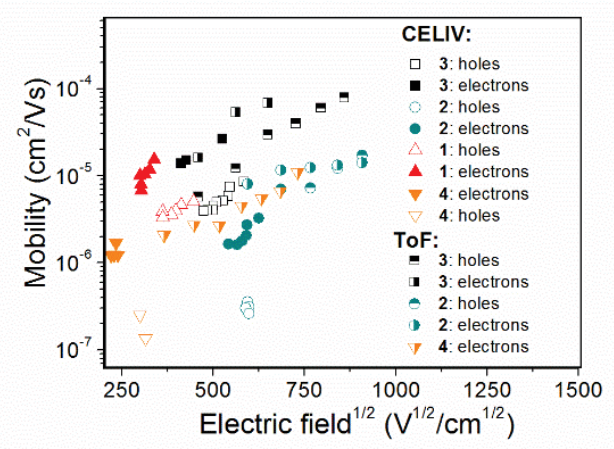

(a)

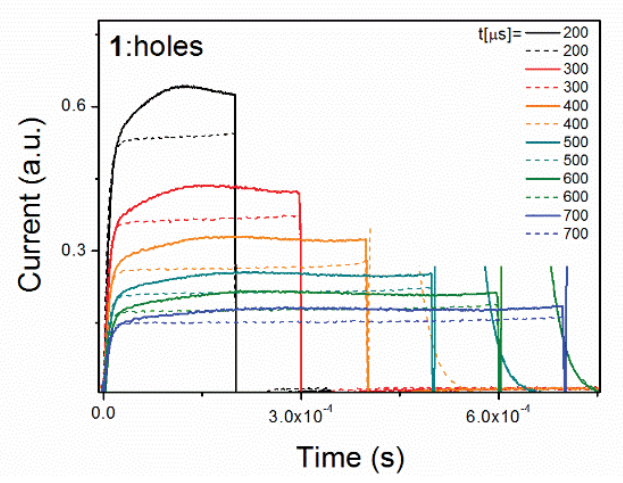

(b)

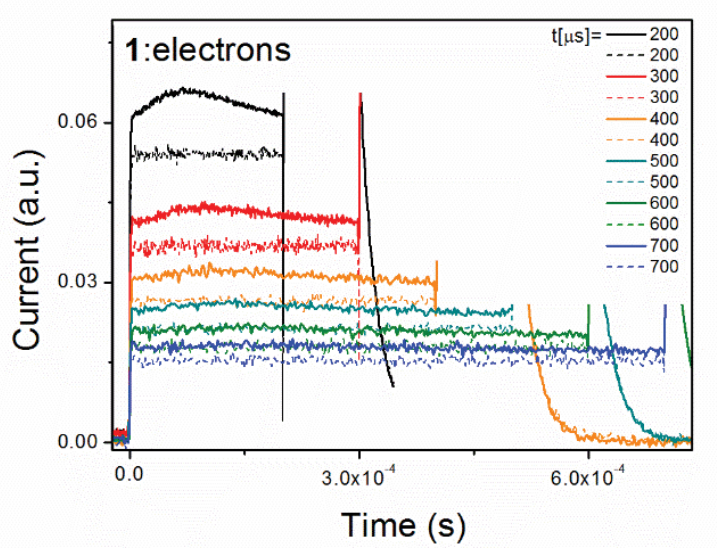


Figure 9. Electric field dependencies of hole and electron drift mobilities measured by TOF and CELIV at room temperature (a); dark-CELIV (dash lines) and photo-CELIV (straight lines) pulses for holes and electrons for the layers of 1-4 (b, c).

\section{Device fabrication and characterization}

OLEDs were fabricated employing $\mathrm{MoO}_{3}$ for the preparation hole injection layer, tris(4carbazoyl-9-ylphenyl)amine (TCTA) or $N, N^{\prime}$-di(1-naphthyl)- $N, \mathrm{~N}^{\prime}$-diphenyl-(1,1'-biphenyl)-4,4'diamine $(\mathrm{NPB})$ for hole transport layer, $(1,3-\mathrm{bis}(N$-carbazolyl)benzene $(\mathrm{mCP})$ for electron blocking layer. The studied compounds (1-4) doped in 3,6-di(9-carbazolyl)-9-(2-ethylhexyl)carbazole (TCz1) or $\mathrm{mCP}$ were used as the emissive material. Diphenyl-4-triphenylsilylphenyl-phosphine oxide (TSPO1) was employed as hole blocking material, 2,2',2"-(1,3,5-benzinetriyl)-tris(1-phenyl-1-Hbenzimidazole) (TPBi) was used for the fabrication of electron transporting layer.

Films of the compounds doped in $\mathrm{mCP}$ had higher PLQY values than molecular mixtures with TCz1. However, TCz1 was considered as preferable host material because of the smaller differences between energy levels of a host and a dopant compounds and better balance of hole and electron mobilities. Two OLEDs were fabricated using compounds $\mathbf{3}$ and $\mathbf{4}$ as emitters. Their structures and energy diagrams are presented in Figure 10. HOMO levels of the emitters were taken from ionization potential values at the first approximation while LUMO levels were estimated by subtracting optical energy gap values of solid state samples from ionization potential values. In OLEDs 3 and 4 compounds 3 and 4 (25 wt\%) doped into TCz1 were used, respectively.Turn-on voltage values for both fabricated devices were found to be low (Table 5). This observation confirms efficient injection from the electrodes and transport of holes and electrons to their recombination sites which is also a reason why electroluminescence spectra indicate emission from the studied compounds only and correspond well with the photoluminescence spectra (see Figures 
2b, 11a). Device 4 emits sky-blue light (CIE coordinates: $0.18,0.32$ ) with an EQE of $6.3 \%$. As it could be expected, device 3 with bipolar TCz1 showed better efficiency (EQE of 6.9\%, luminous efficacy of $12.9 \mathrm{~lm} / \mathrm{W}$ ) than $\mathrm{mCP}$-based device with $\mathrm{mCP}$ as a host and compound $\mathbf{3}$ as emitter (EQE of $5.6 \%, 10.4 \mathrm{~lm} / \mathrm{W}$ Figures S24-S26). However, the difference of emitting colours is negligible (CIE coordinates: $0.28,0.52$ for 3, 0.28, 0.50 for mCP-based device) which is the evidence of the well-designed structures of OLEDs.

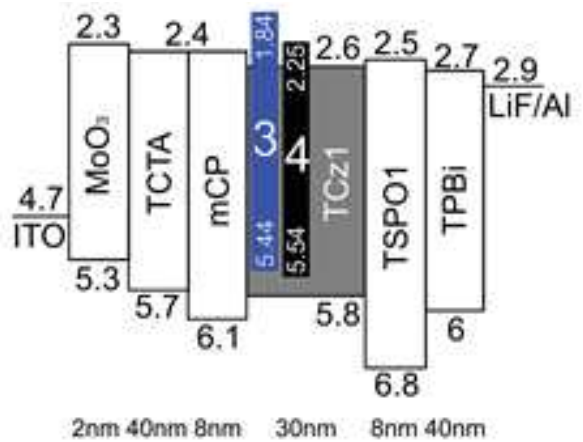

Figure 10. Energy diagrams of devices 3 and 4.

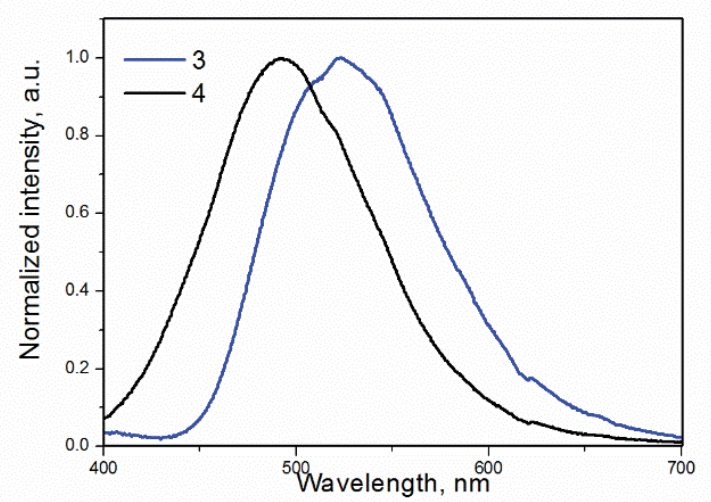

(a) 


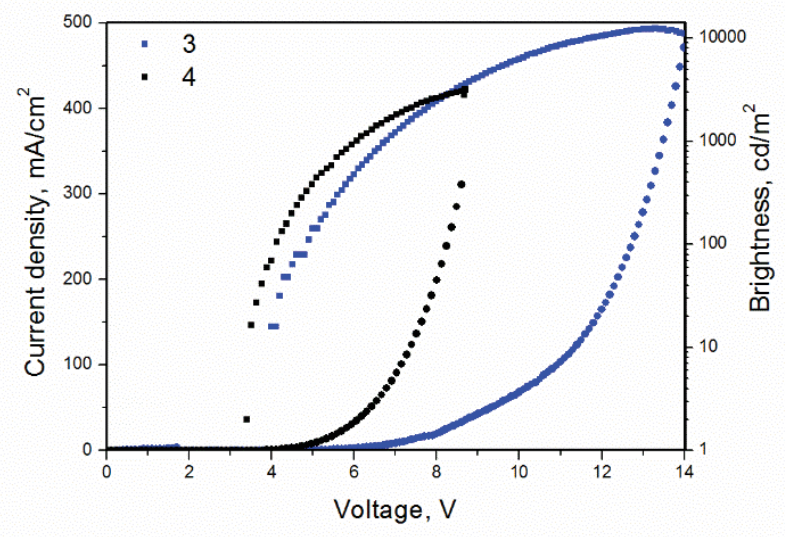

(b)

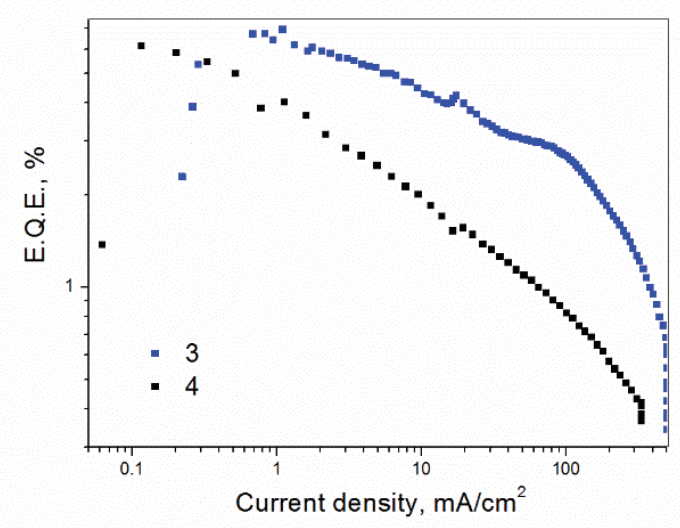

(c)

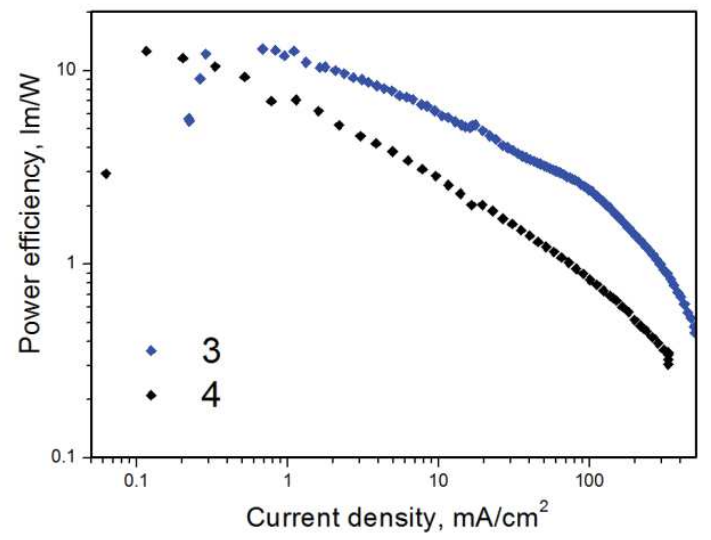

(d) 
Figure 11. Electroluminescence characteristics of OLEDs based on the emitter 3 or 4: (a) normalized electroluminescence spectra recorded at 6V; (b) luminance, current density; c) external quantum efficiency; d) power efficiency.

Table 5. Photophysical parameters derived from steady state and time resolved spectroscopic measurements of dilute THF solutions and thin films of compounds 1-4 at 77 $\mathrm{K}$ and at room temperature.

\begin{tabular}{ccccccc} 
Comp. & $\begin{array}{c}\Phi_{\mathrm{PL} \mathrm{TC1} 1} \\
{[\mathrm{a}], \%}\end{array}$ & $\begin{array}{c}\Phi_{\mathrm{PL} \mathrm{mCP}} \\
{[\mathrm{b}], \%}\end{array}$ & $\begin{array}{c}\Phi_{\mathrm{PL} \text { neat }} \\
{[\mathrm{c}], \%}\end{array}$ & $\mathrm{E}_{\mathrm{S} 1}{ }^{\mathrm{dd}]}, \mathrm{eV}$ & $\mathrm{E}_{\mathrm{T} 1}{ }^{[\mathrm{d}]}, \mathrm{eV}$ & $\Delta \mathrm{E}_{\mathrm{ST}}{ }^{[\mathrm{d}]}, \mathrm{eV}$ \\
\hline $\mathbf{1}$ & 10.9 & 21.8 & 24.4 & $3.49(3.01)$ & $3.05(2.84)$ & $0.44(0.17)$ \\
\hline $\mathbf{2}$ & 8.2 & 11.6 & 9.2 & $2.65(2.24)$ & $2.64(2.22)$ & $0.01(0.02)$ \\
\hline $\mathbf{3}$ & 20.8 & 32.1 & 11.9 & $2.84(2.17)$ & $2.78(2.14)$ & $0.06(0.02)$ \\
\hline $\mathbf{4}$ & 23.9 & 36.5 & 33.1 & $2.97(2.53)$ & $2.92(2.50)$ & $0.05(0.02)$
\end{tabular}

[a] PLQY of the films of compounds doped in TCz1 (25wt\%). [b] PLQY of the films of compounds doped in mCP $(5 \mathrm{wt} \%)$. [c] PLQY of the neat films. [d] Estimated from the PL spectra of the dilute THF solutions at $77 \mathrm{~K}$. The theoretical values of $\mathrm{E}_{\mathrm{S} 1}, \mathrm{E}_{\mathrm{T} 1}$ and $\Delta \mathrm{E}_{\mathrm{ST}}$ are presented in parentheses.

For the optimized structure of OLEDs with non-doped layer of the $4 \mathrm{Wu}$ et $\mathrm{al}^{24}$. observed EQE of $17 \%$. Considering our results compound 3 containing phenoxazine moiety is very promising as emitter for highly efficient OLEDs.

\section{Conclusions}

Diphenylsulfone derivatives having acridine, carbazole, phenothiazine and phenoxazine moieties as donor unites at meta positions of diphenylsulfone moieties were synthesized and investigated. The differences in properties were justified by the theoretical study using DFT and TD-DFT calculations. Dilute toluene solutions of compounds with acridan and phenoxazine as donors exhibited small singlet-triplet energy differences and up to 4.7 times higher intensities of photoluminescence of the solutions after degassing. Photoluminescence decay curves of the solid samples of the compounds of their molecular mixtures with high-triplet energy hosts had 
components in $\mu$ s range. Ionization potential values estimated by cyclic voltammetry ranged from 5.05 to $5.80 \mathrm{eV}$ and electron affinity values ranged from 1.67 to $2.44 \mathrm{eV}$. Solid-state ionization potentials estimated by photoelectron emission spectrometry ranged from 5.6 to $5.9 \mathrm{eV}$. The compounds exhibited well-balanced hole and electron mobilities exceeding $10^{-5} \mathrm{~cm}^{2} / \mathrm{V} \cdot \mathrm{s}$ at an electric field higher than $2.5 \cdot 10^{5} \mathrm{~V} / \mathrm{cm}$. Sky-blue and green efficient OLEDs based compounds containing acridan and phenoxazine as TADF emitters were fabricated. The devices showed maximum brightness of 3200 and $12300 \mathrm{~cd} / \mathrm{m}^{2}$ and maximum external quantum efficiency of 6.3 and $6.9 \%$, respectively.

\section{Acknoledgmments}

This research was funded by the European Social Fund according to the activity 'Improvement of researchers' qualification by implementing world-class R\&D projects' of Measure No. 09.3.3-LMT-K-712. DG acknowledges to the ERDF PostDoc project No. 1.1.1.2/VIAA/1/16/177.

\section{References}

[1] Q. Zhang, Q. Zhou, Y. Cheng, L. Wang, D. Ma, X. Jing and F. Wang, Adv. Mater., 2004, 16, 432 .

[2] H. Uoyama, K. Goushi, K. Shizu, H. Nomura and C. Adachi, Nature, 2012, 492, 234.

[3] A. Chihaya, Jpn. J. Appl. Phys., 2014, 53, 60101.

[4] K. K. Rohatgi-Mukherjee, New Delhi, India: Wiley Eastern Limited, 1978, p. 105. 
[5] F. B. Dias, K. N. Bourdakos, V. Jankus, K. C. Moss, K. T. Kamtekar, V. Bhalla, J. Santos, M. R. Bryce, and A. P. Monkman, Adv. Mater.,2013, 25, 3707.

[6] M. A. Baldo, C. Adachi and S. R. Forrest, Phys. Rev. B:Condens. Matter Mater. Phys., 2000, 62, 10967.

[7] M.K. Etherington, J. Gibson, H.F. Higginbotham, T.J. Penfold and A.P. Monkman, Nat Commun. 2016, 7, $136801-7$.

[8] Q. Zhang, J. LI, K. Shizu, S. Huang, S. Hirata, H. Miyazaki and C. Adachi, J. Am. Chem. Soc., 2012, 134, 14706.

[9] Z. Yang, Z. Mao, Z. Xie, Y. Zhang, S. Liu, J. Zhao, J. Xu, Z. Chi and M. P. Aldred, Chem. Soc. Rev., 2017, 46, 915-1016.

[10] B. Huang, Q. Qi, W. Jiang, J. Tang, Y. Liu, W. Fan, Z. Yin, F. Shi, X. Ban, H. Xu and Y. Sun, Dyes Pigm., 2014, 111, 135-144.

[11] D. Gudeika, D. Volyniuk, J. V. Grazulevicius, E. Skuodis, S. Y. Yu, W. T. Liou, L. Y. Chen and Y. J. Shiu, Dyes Pigm., 2016, 130, 298-305.

[12] E. Miyamoto, Y. Yamaguchi, M. Yokoyama, Electrophotography 1989, 28, 364-370.

[13] D. Gudeika, J.V. Grazulevicius, D. Volyniuk, G. Juska, V. Jankauskas and G. Sini. J. Phys. Chem. C, 2015, 119 (51), 28335-28346.

[14] a) V. Mimaite, J.V. Grazulevicius, R. Laurinaviciute, D. Volyniuk, V. Jankauskas and G. Sini, J. Mater. Chem. C, 2015, 3, 11660-11674; b) D. Gudeika, G. Sini, V. Jankauskas, G. Sych and J. V. Grazulevicius, RSC Advances, 2016, 6, 2191-2201.

[15] A. Pivrikas, N.S. Sariciftci, G. Juska, R. Osterbacka, Prog. Photovolt: Res. Appl. 2007, $15,677-696$.

[16] P.L. Santos, J.S. Ward, P. Data, A.S. Batsanov, M.R. Bryce, F.B. Dias and A.P. Monkman, J. Mater. Chem. C, 2016, 4, 3815-3824. 
[17] D. Gudeika, D. Volyniuk, V. Mimaite, R. Lytvyn, R. Butkute, O. Bezvikonnyi, G. Buika and J. V. Grazulevicius, Dyes Pigm., 2017, 142, 394-405.

[18] E. Skuodis, A. Tomkeviciene, R. Reghu, L. Peciulyte, K. Ivaniuk, D. Volyniuk, O. Bezvikonnyi, G. Bagdziunas, D. Gudeika and J. V. Grazulevicius, Dyes Pigm., 2017, $139,795-807$.

[19] T. Förster, Discuss. Faraday Soc., 1959, 27,7-17.

[20] M. Y. Wong and E. Zysman-Colman, Adv. Mater., 2017, 29, 1605444.

[21] K. Shanmugasundaram, M. S. Subeesh, C. D. Sunesh, R. K. Chitumalla, J. Jang, and Y. Choe, J. Phys. Chem. C, 2016, 120 (36), 20247-20253.

[22] D. L. Dexter, J. Chem. Phys., 1953, 21, 836-850.

[23] K. Wu, Z. Wang, L. Zhan, C. Zhong, S. Gong, G. Xie, and C. Yang, J Phys Chem Lett., 2018, 9(7), 1547-1553.

[24] Q. Zhang, J. Li, K. Shizu, S. Huang, S. Hirata, H. Miyazaki and C. Adachi, J. Am. Chem. Soc., 2012, 134, 14706-14709.

[25] F. B. Dias, K. N. Bourdakos, V. Jankus, K. C. Moss, K. T. Kamtekar, V. Bhalla, J. Santos, M. R. Bryce and A. P. Monkman, Adv. Mater., 2013, 25, 3707-3714.

[26] T. Chen, L. Zheng, J. Yuan, Z. An, R. Chen, Y. Tao, H. Li, X. Xie and W, Huang, Sci. Rep., 2015, 5, 10923.

[27] F. B. Dias, T. J. Penfold and A. P. Monkman, Methods Appl. Fluoresc., 2017, 5, 012001.

[28] B.W. D’Andrade S. Datta, S.R. Forrest, P. Djurovich, E. Polikarpov and M.E. Thompson, Org Electron, 2005, 6, 11-20.

[29] P. Politzer and F. Abu-Awwad, Theor. Chim. Acta, 1998, 99, 83-87. 


\title{
Effect of donor substituents on thermally activated delayed fluorescence of diphenylsulfone derivatives
}

\author{
Oleksandr Bezvikonnyi, ${ }^{\text {a,b }}$ Dalius Gudeika, ${ }^{\text {a,c }}$ Dmytro Volyniuk, ${ }^{\text {a }}$ Viktorija Mimaite, ${ }^{\text {a }}$ \\ Bernard Ronit Sebastine ${ }^{\mathrm{a}}$ and Juozas V. Grazulevicius ${ }^{\mathbf{a}^{*}}$
}

aDepartment of Polymer Chemistry and Technology, Kaunas University of Technology, Radvilenu pl. 19, LT-50254, Kaunas, Lithuania

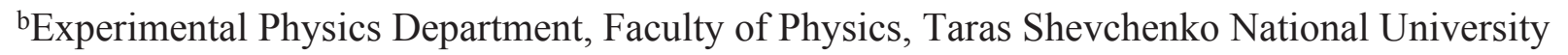
of Kyiv, pros. Akademika Glushkova 4b, Kyiv, Ukraine

c Institute of Solid State Physics, University of Latvia, 8 Kengaraga St., Riga LV-1063, Latvia

* Corresponding author: juozas.grazulevicius@ktu.lt 


\section{Supplementary data}

\section{Tables}

Table S1. PL lifetimes of the films of derivatives doped in $\mathrm{mCP} 5 \mathrm{wt} \%$, in $\mathrm{TCz} 125 \mathrm{wt} \%$ and neat thin films of compounds detected at room temperature

\begin{tabular}{c|c|c|c} 
compound & doped in mCP 5wt \% film & doped in TCz1 25wt\% & neat thin films \\
\hline 1 & $0.5 \mathrm{~ns}, 6.67 \mathrm{~ns}$ and $20.04 \mathrm{~ns}$ & $4.8 \mathrm{~ns}, 18.81 \mathrm{~ns}$ and $81.53 \mathrm{~ns}$ & $7.57 \mathrm{~ns}$ and $24.88 \mathrm{~ns}$ \\
\hline 2 & $4.21 \mathrm{~ns}, 17.11 \mathrm{~ns}$ and $37.75 \mathrm{~ns}$ & $3.47 \mathrm{~ns}, 15.51 \mathrm{~ns}$ and $34.94 \mathrm{~ns}$ & $28.96 \mathrm{~ns}$ and $1310 \mathrm{~ns}$ \\
\hline 3 & $48.27 \mathrm{~ns}$ and $3851.88 \mathrm{~ns}$ & $32.47 \mathrm{~ns}$ and $866.58 \mathrm{~ns}$ & $58.86 \mathrm{~ns}$ and 987.57 \\
\hline 4 & $46.04 \mathrm{~ns}$ and $772.97 \mathrm{~ns}$ & $40.86 \mathrm{~ns}$ and $190.46 \mathrm{~ns}$ & $22.31 \mathrm{~ns}, 67.74 \mathrm{~ns}$ and $1340.06 \mathrm{~ns}$
\end{tabular}

\section{Figures}

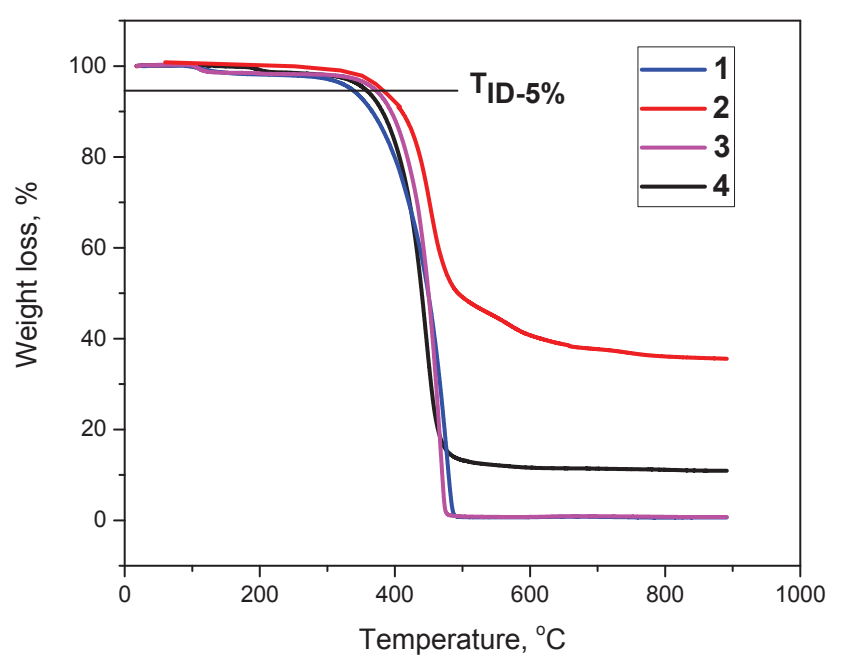

Figure S1. TGA curves of 1-4. 

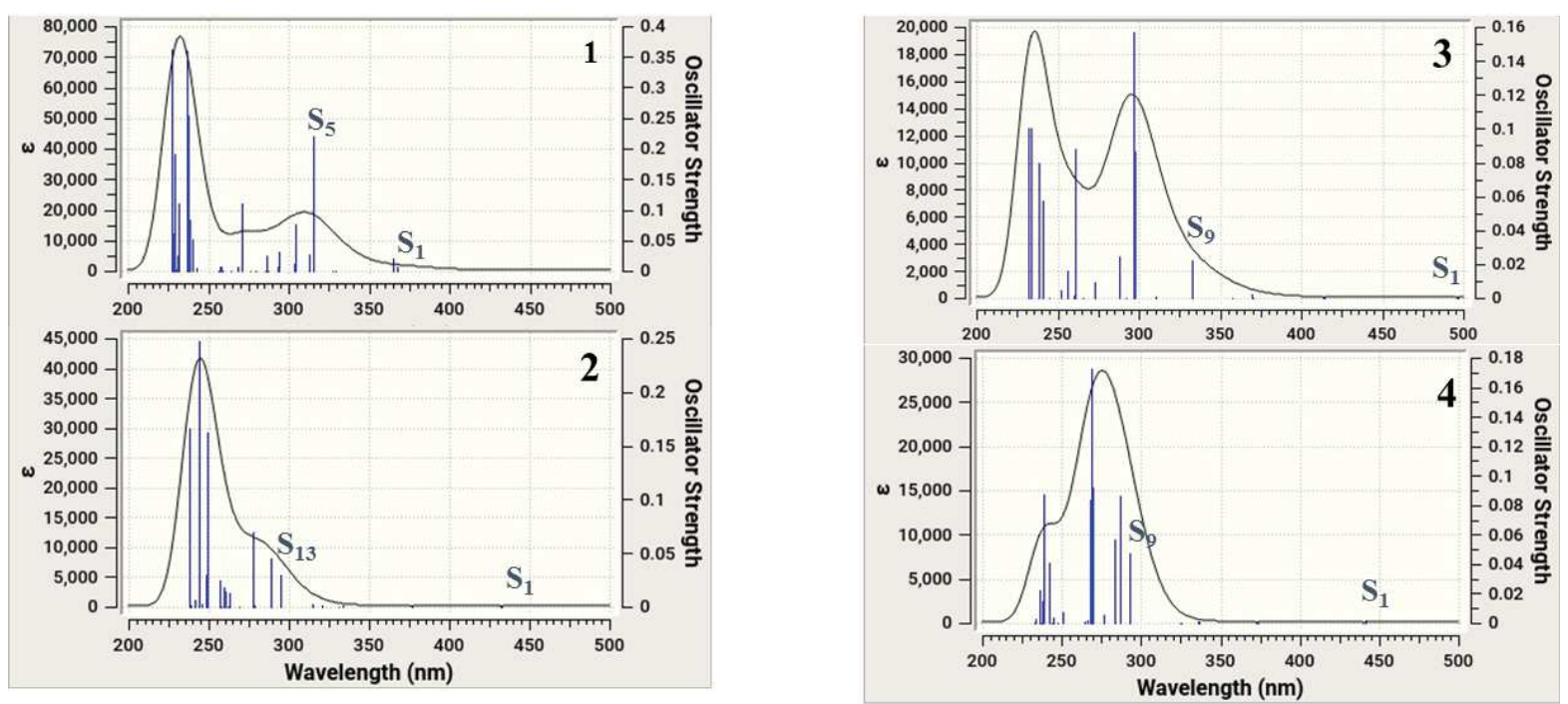

Figure S2. The theoretical spectra of $\mathbf{1 - 4}$ were obtained by mean of TD-DFT calculations (gas phase). The absorption bands are obtained by considering peak half-widths at half height of 0.3

$\mathrm{eV}$

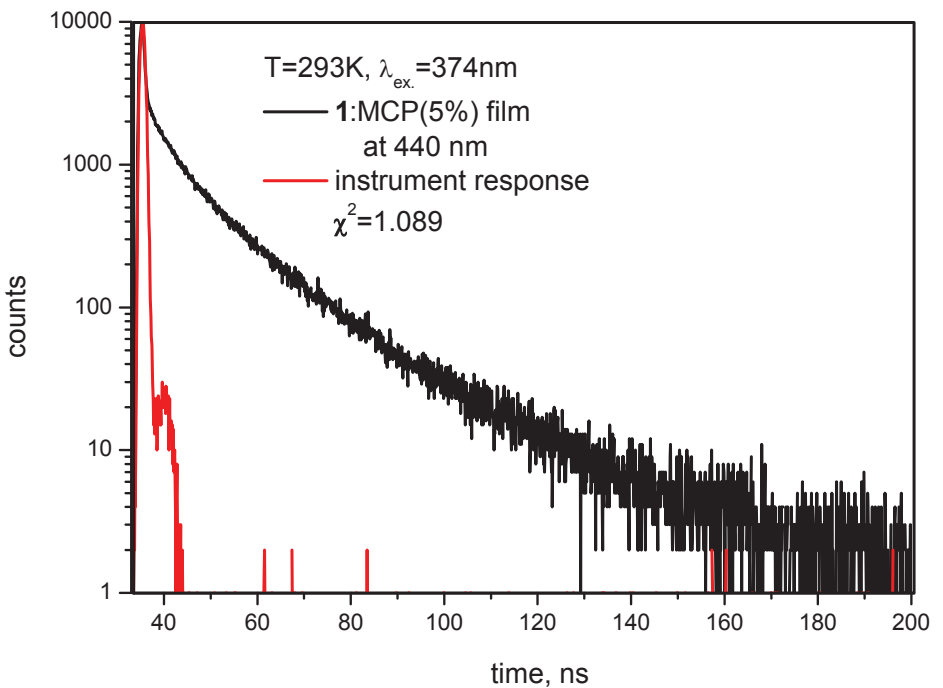

Figure S3. Photoluminescence decay curve of the film of $\mathbf{1}$ doped in $\mathrm{mCP} 5 \mathrm{wt} \%$. 


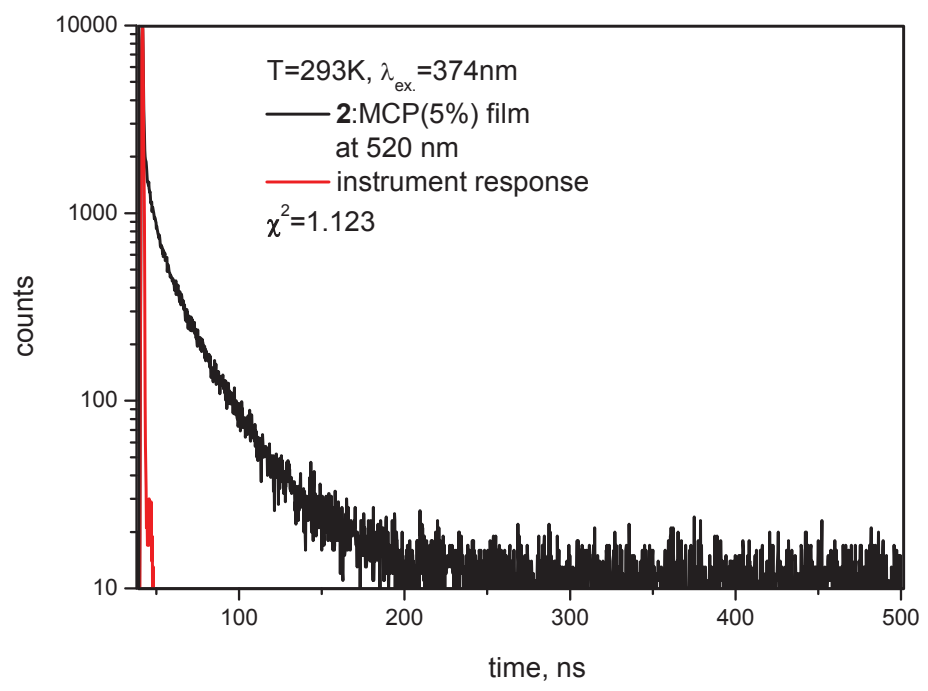

Figure S4. Photoluminescence decay curve of the film of 2 doped in $\mathrm{mCP} 5 \mathrm{wt} \%$.

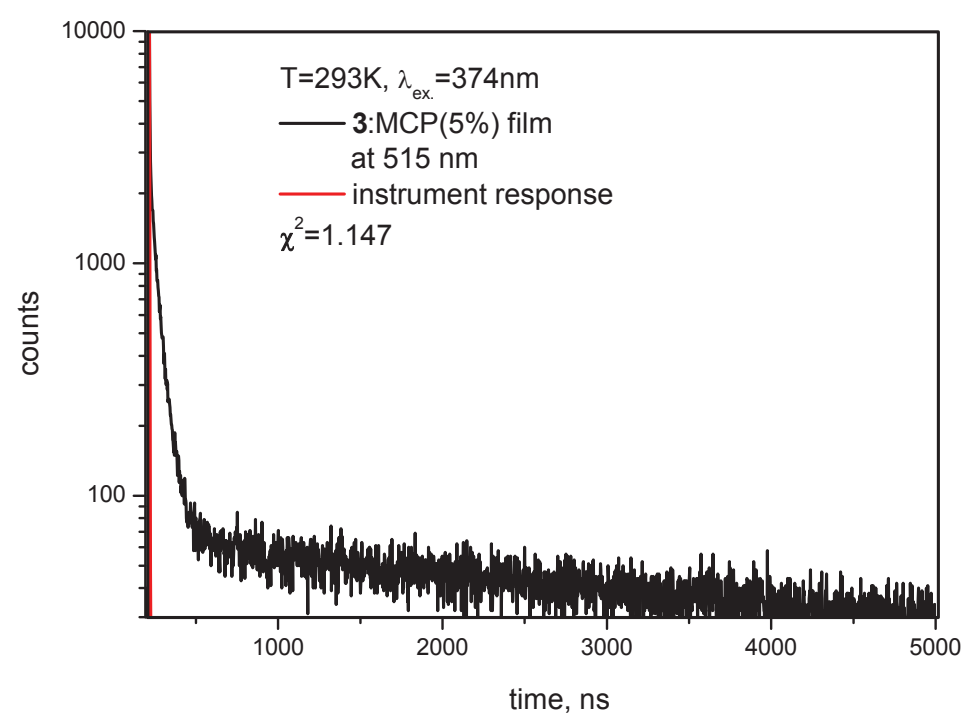

Figure S5. Photoluminescence decay curve of the film of 3 doped in mCP 5wt\%. 


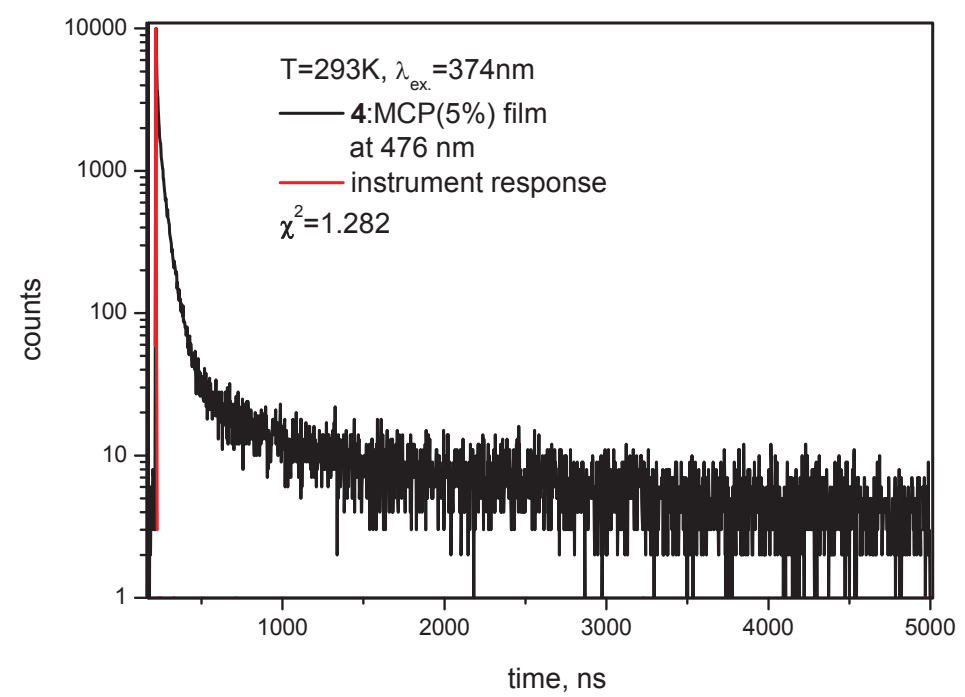

Figure S6. Photoluminescence decay curve of the film of 4 doped in $\mathrm{mCP} 5 \mathrm{wt} \%$.

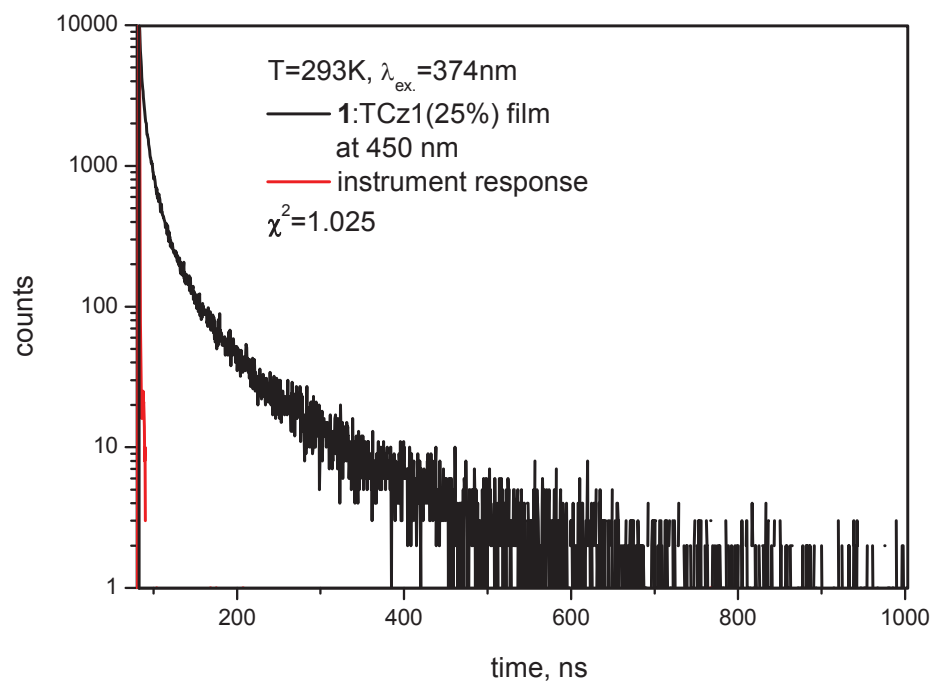

Figure S7. Photoluminescence decay curve of the film of 1 doped in TCz1 $25 \mathrm{wt} \%$. 


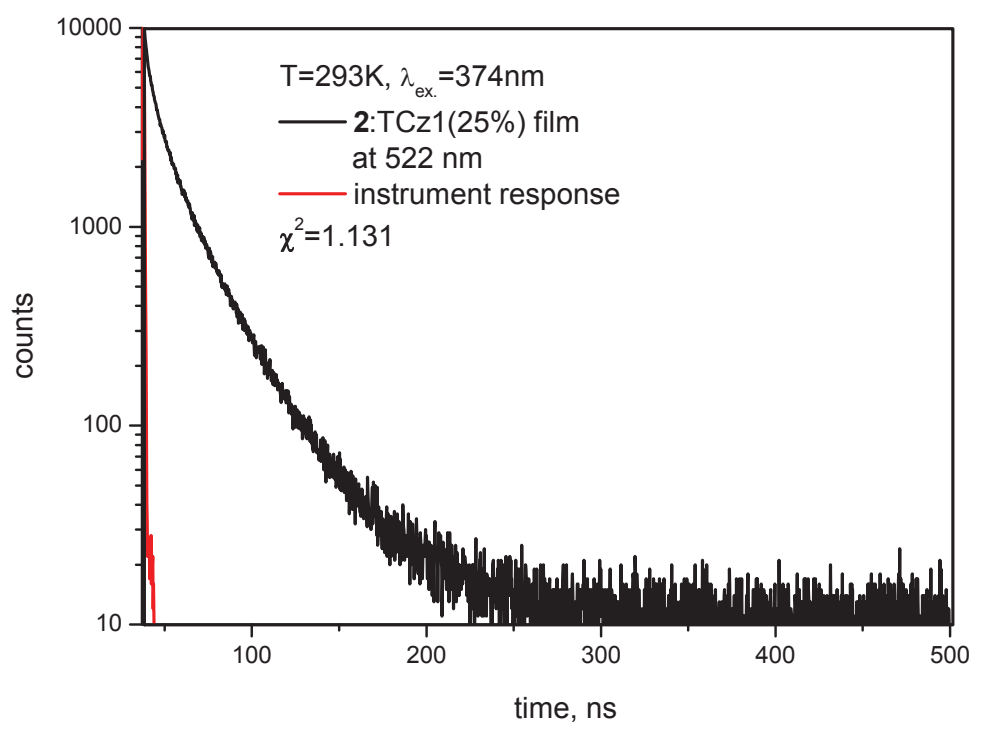

Figure S8. Photoluminescence decay curve of the film of $\mathbf{2}$ doped in TCz1 25wt $\%$.

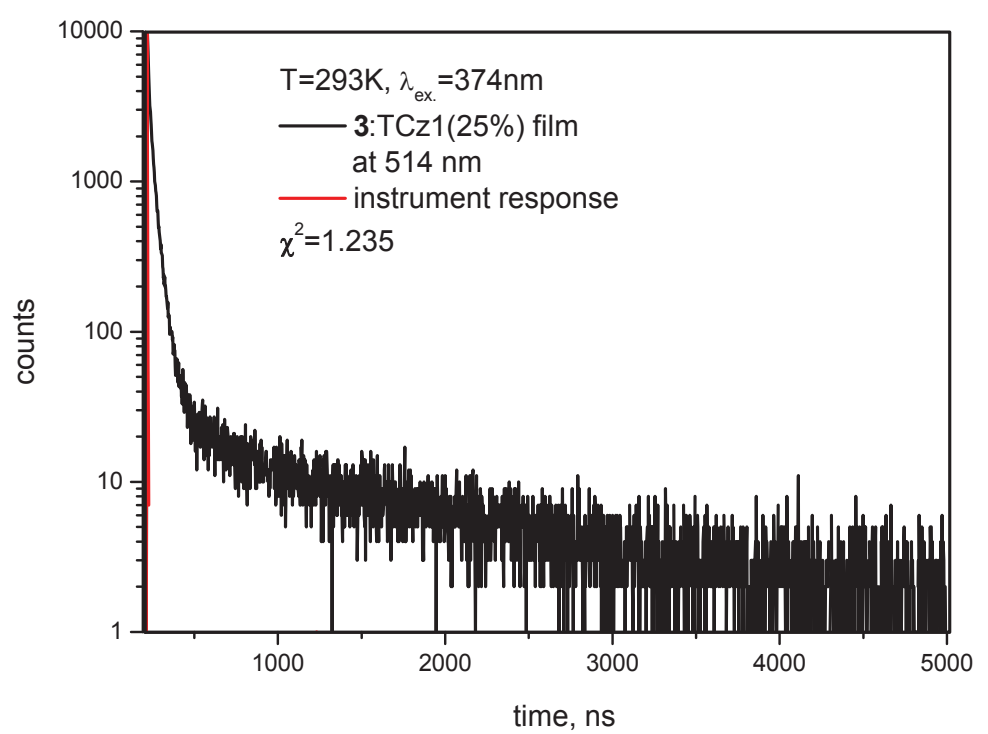

Figure S9. Photoluminescence decay curve of the film of $\mathbf{3}$ doped in TCz1 $25 \mathrm{wt} \%$. 


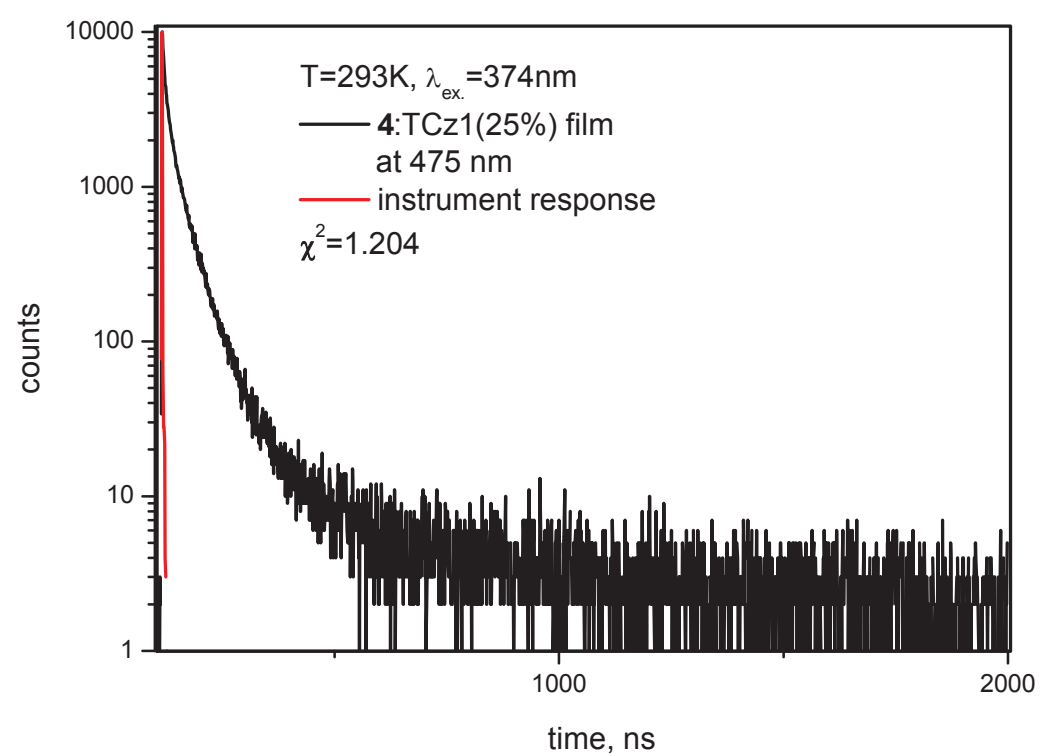

Figure S10. Photoluminescence decay curve of the film of 4 doped in TCz1 25wt $\%$.

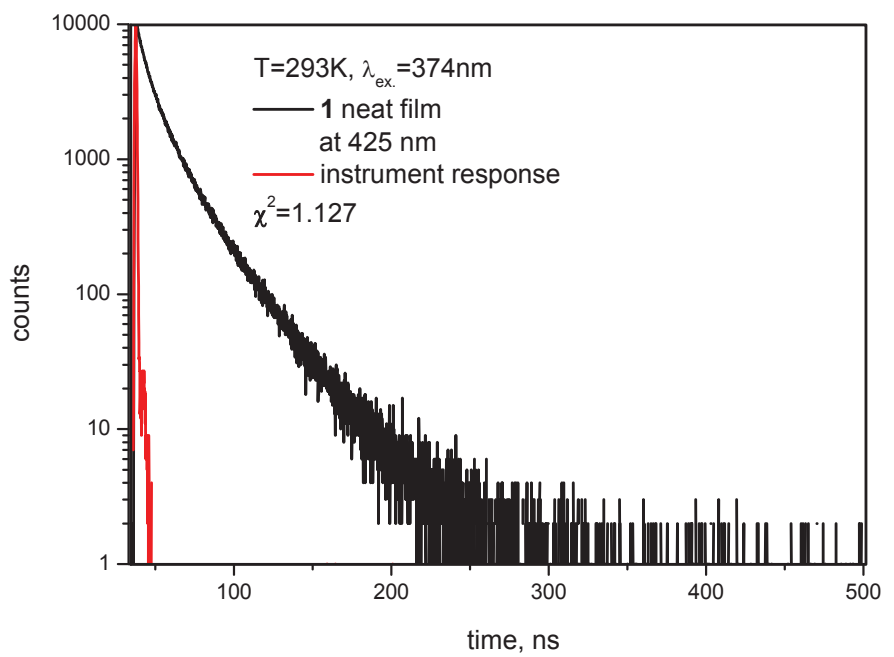

Figure S11. Photoluminescence decay curve of the neat film of $\mathbf{1 .}$ 


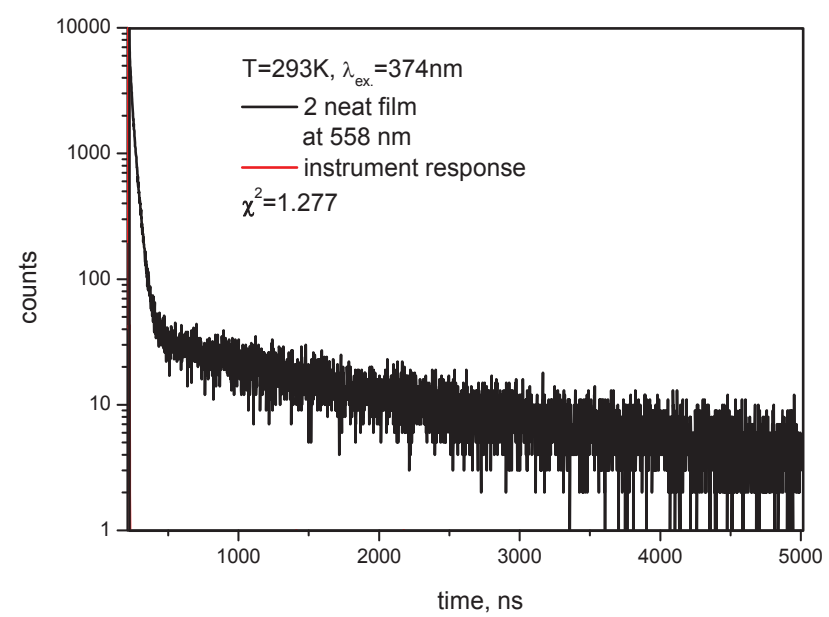

Figure S12. Photoluminescence decay curve of the neat film of 2.

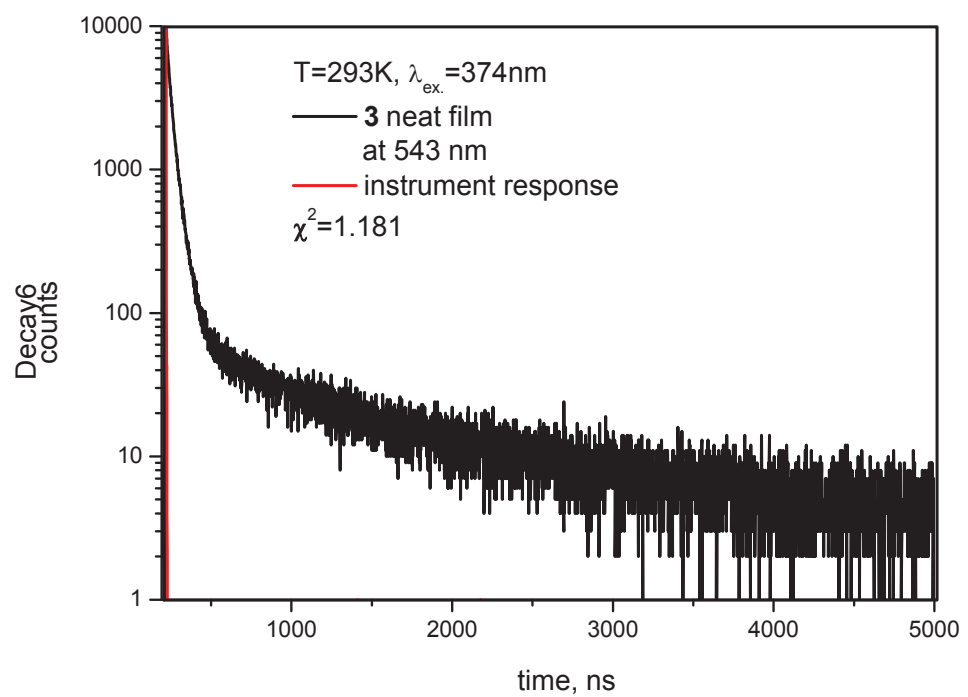

Figure S13. Photoluminescence decay curve of the neat film of $\mathbf{3}$. 


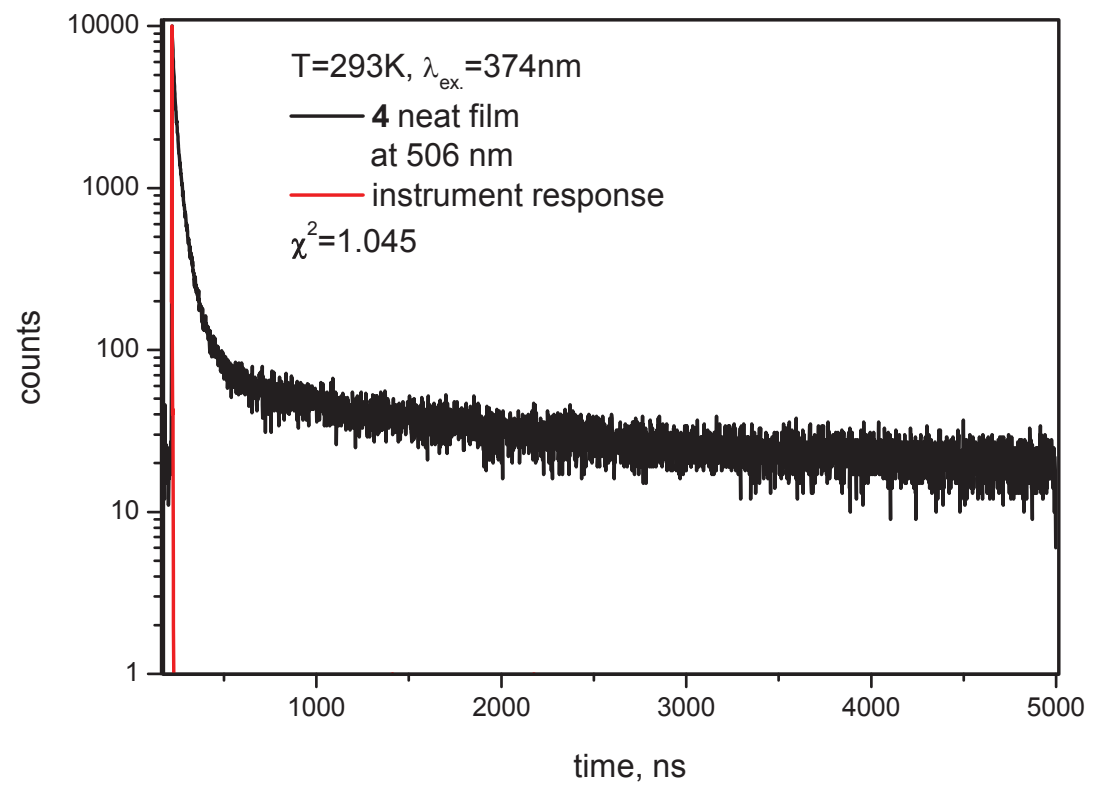

Figure S14. Photoluminescence decay curve of the neat film of 4.

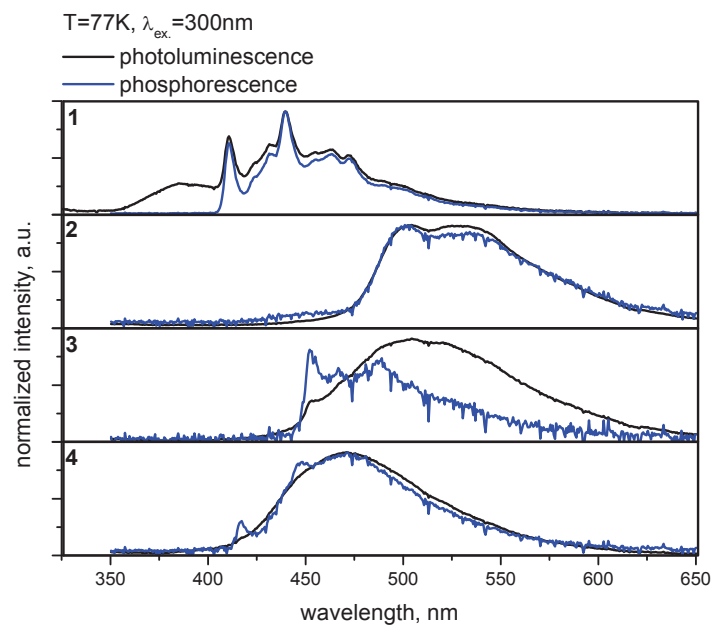

Figure S15. PL and phosphorescence spectra of dilute THF solutions at 77K. 


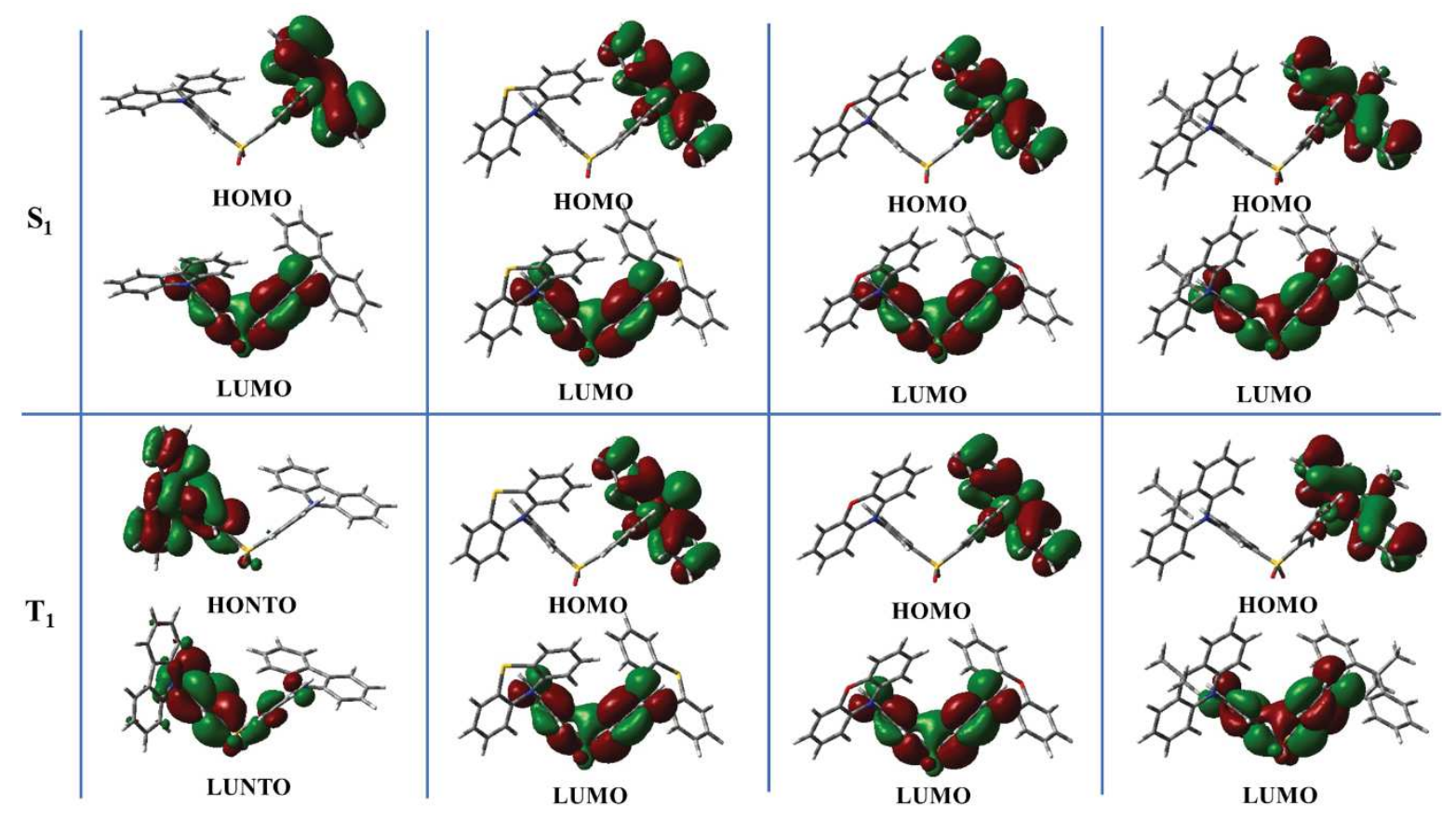

Figure S16. The molecular orbitals in $\mathrm{S}_{1}$ and $\mathrm{T}_{1}$ excited state geometry for 1-4 calculated at TDDFT. NTO was used for the visualization of HONTO and LUNTO

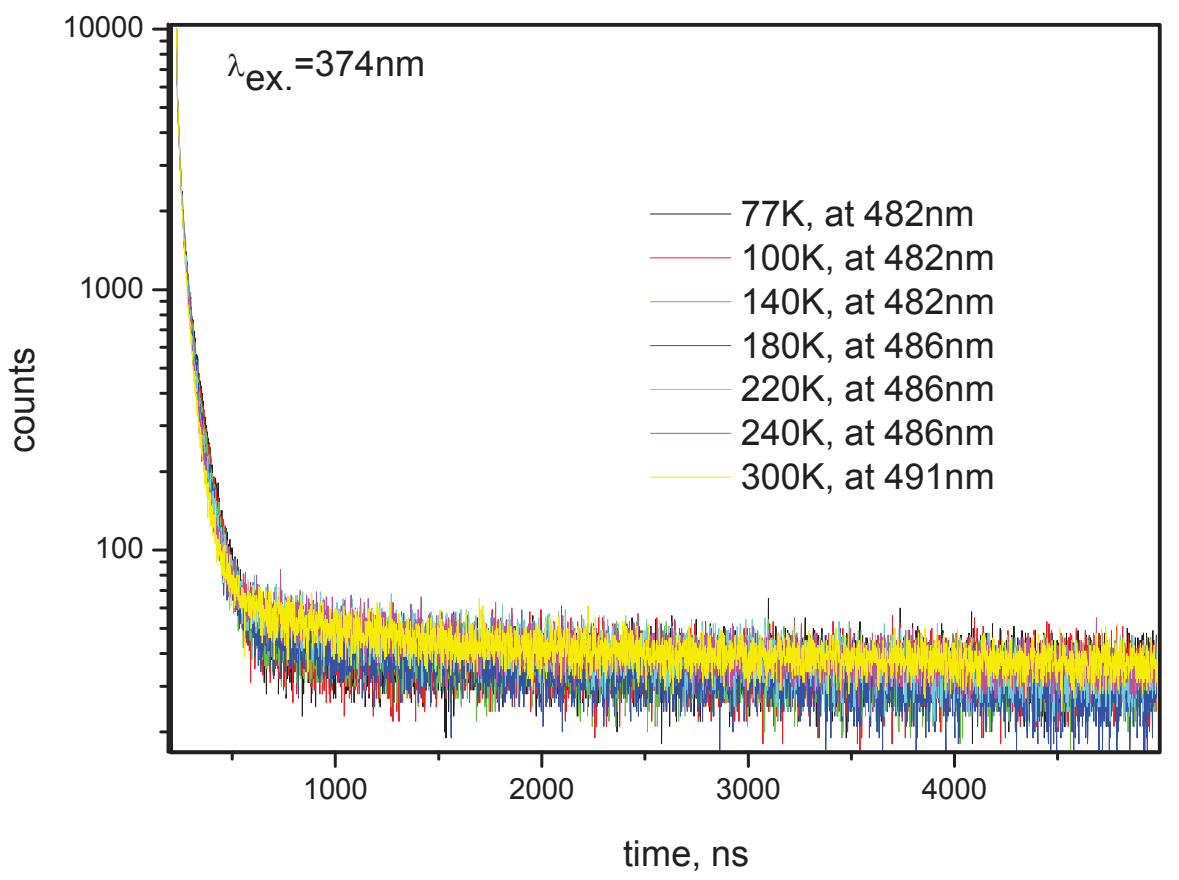

Figure S17. Photoluminescence decay curves of the film of $\mathbf{4}$ doped in TCz1 $25 \mathrm{wt} \%$ at different temperatures. 


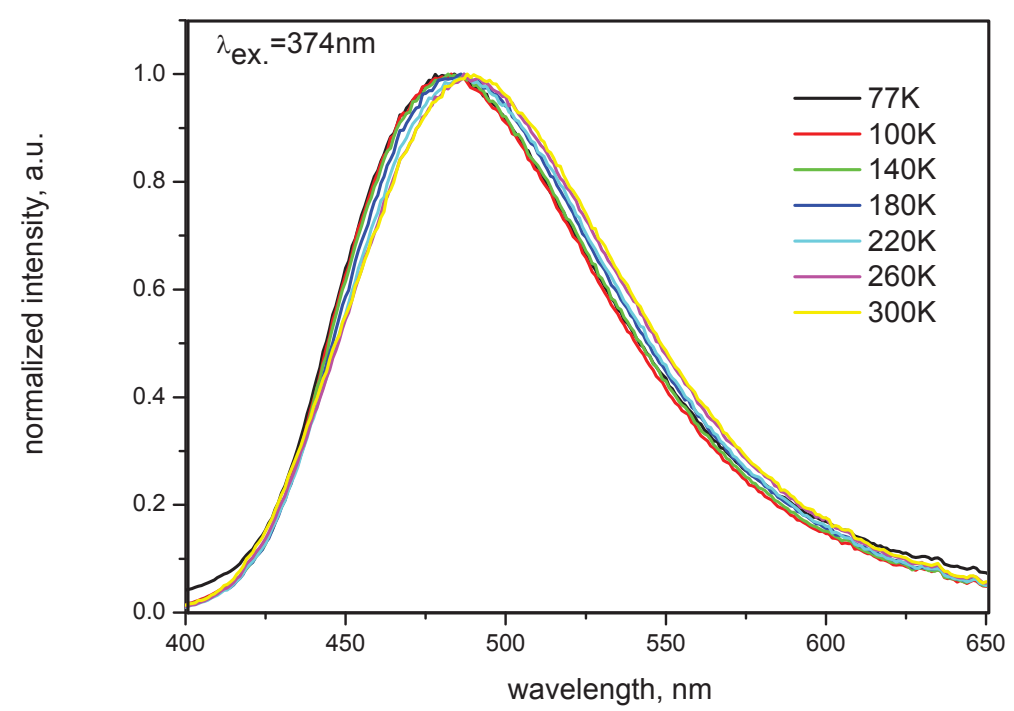

Figure S18. Photoluminescence spectra of the film of 4 doped in TCz1 $25 \mathrm{wt} \%$ at different temperatures.

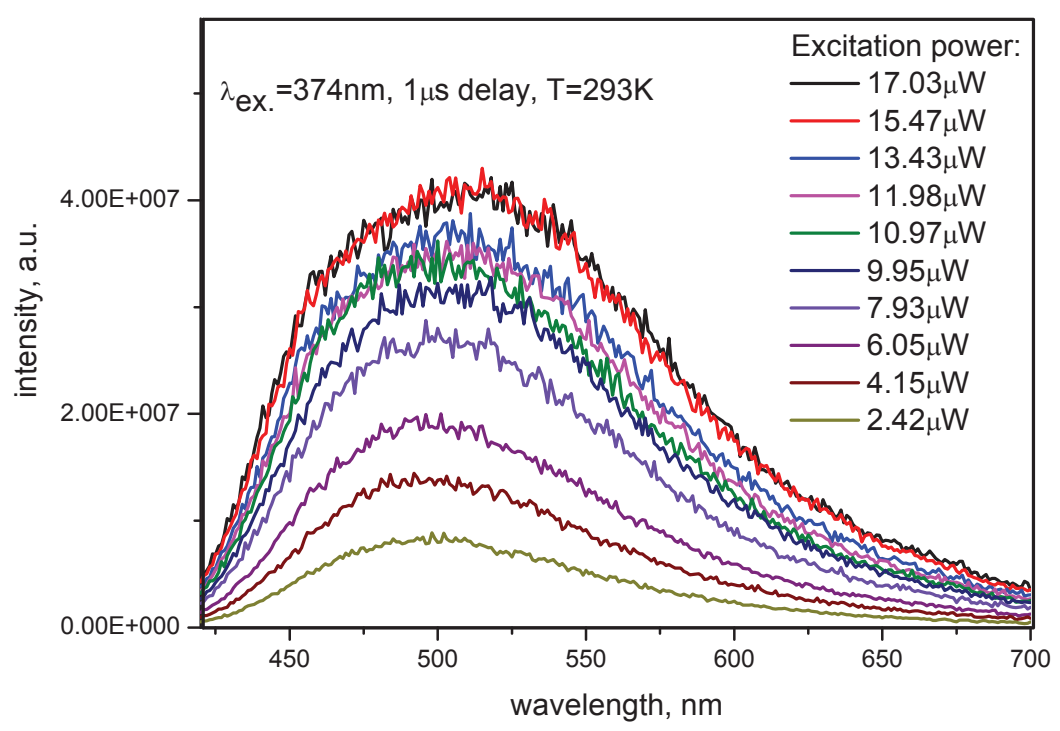

Figure S19. Power dependence of the DF of the film of 4 doped in TCz1 $25 \mathrm{wt} \%$. 


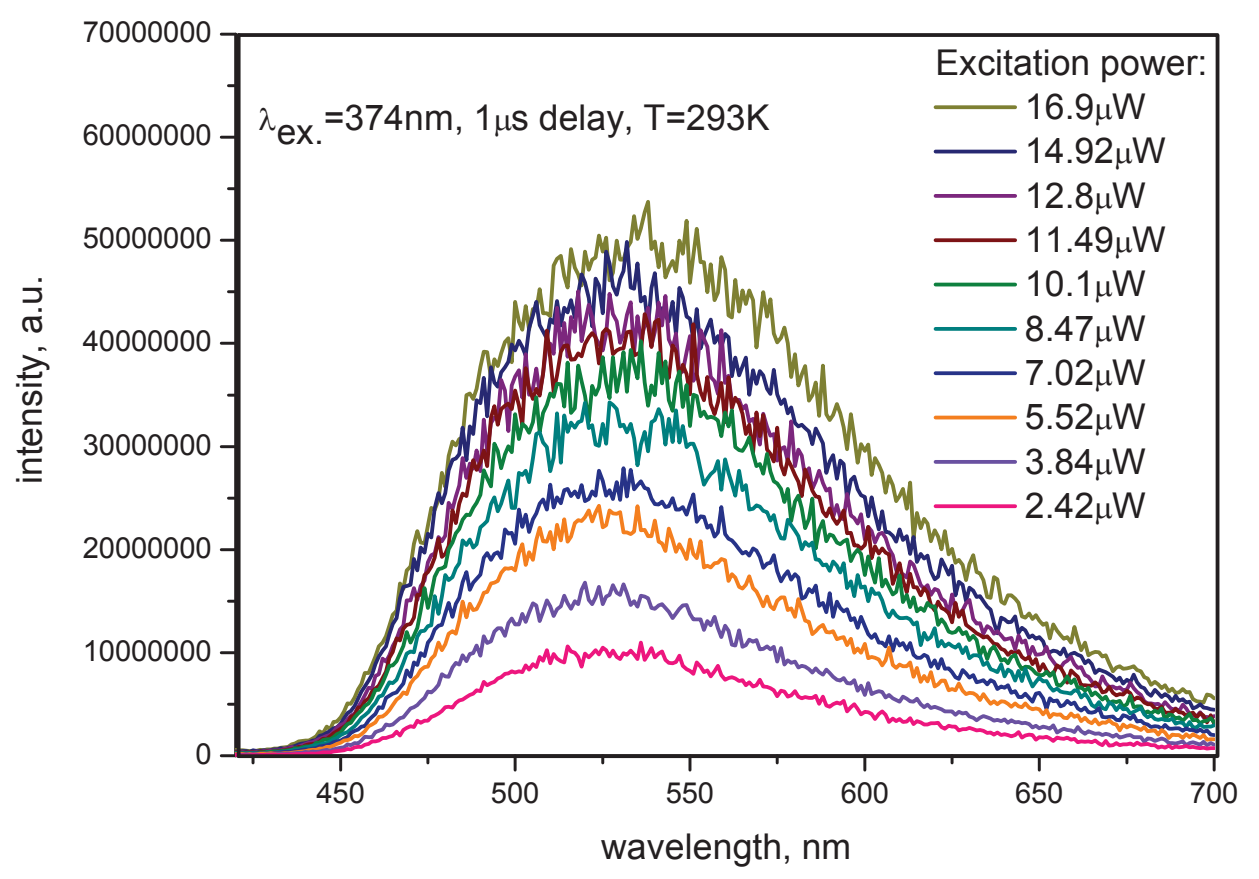

Figure S20. Power dependence of the DF of the film of 3 doped in TCz1 $25 \mathrm{wt} \%$.

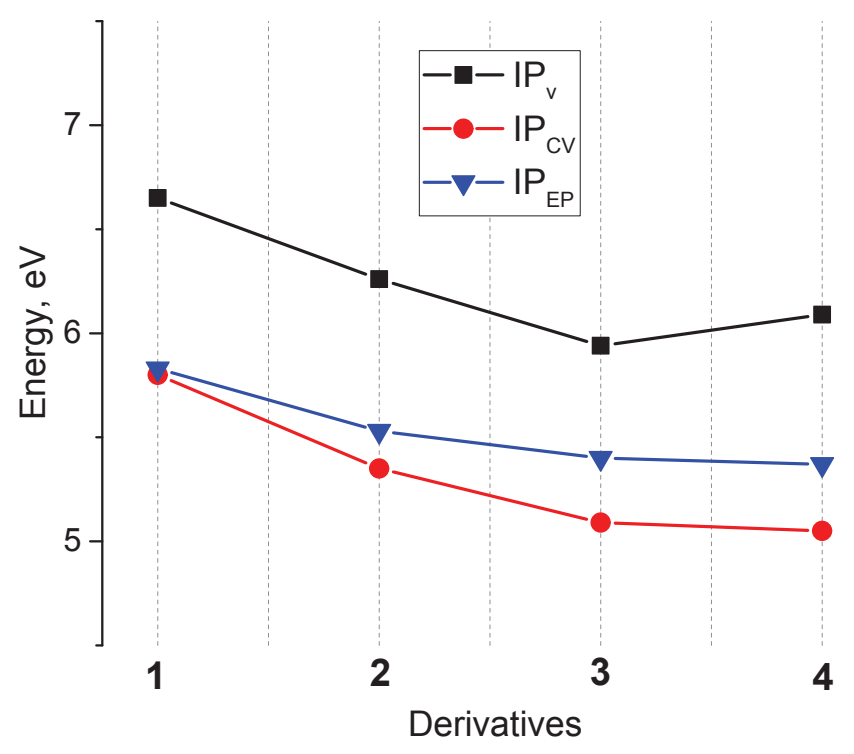

Figure S21. Graphical comparison of vertical ionization potentials $\left(\mathrm{IP}_{\mathrm{v}}\right)$, calculated at the B3LYP/6-31G(d) level of theory, electrochemical IP values and ionization potentials of the solid layers of 1-4 estimated by electron photoemission method in air. 

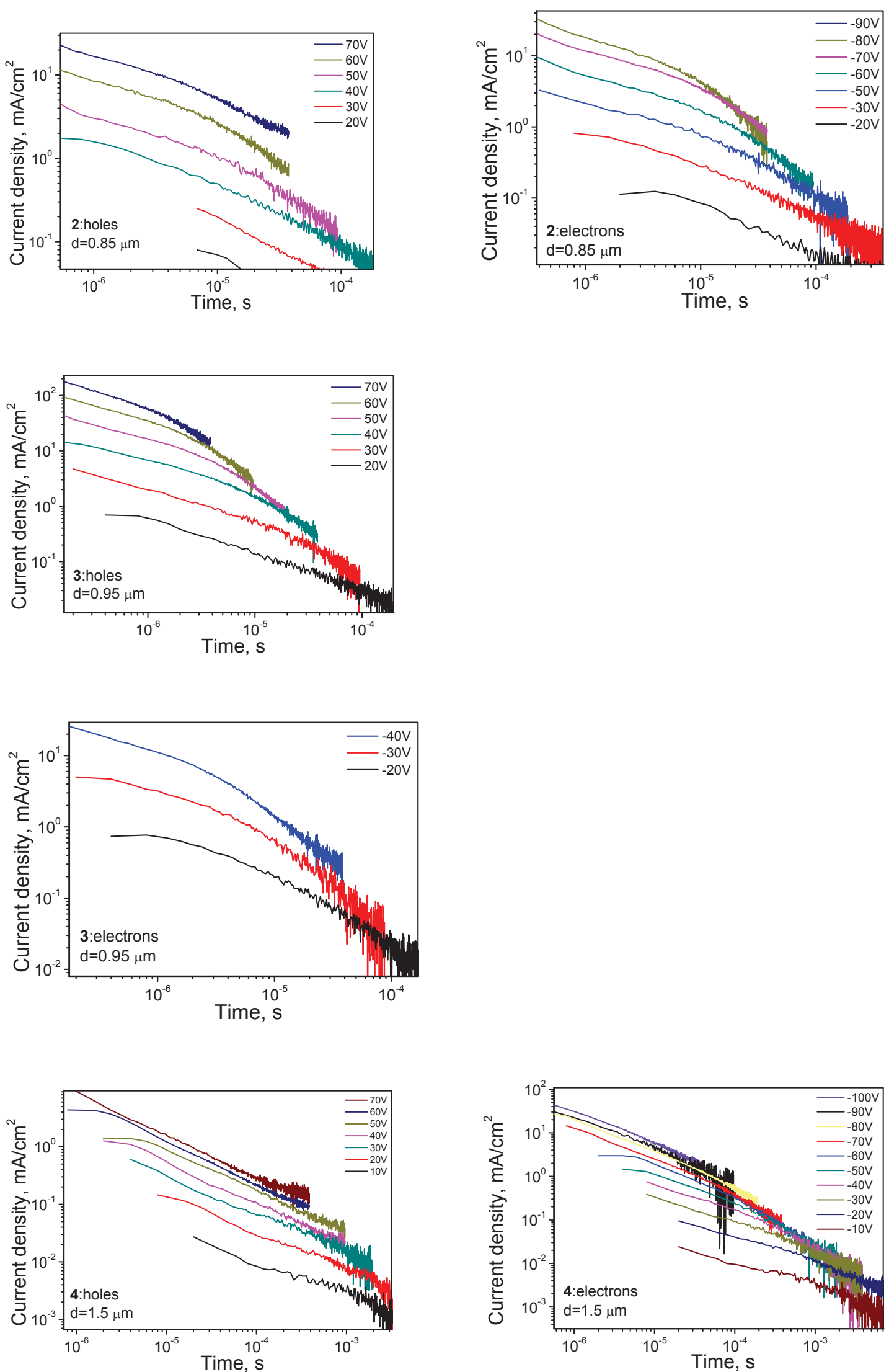

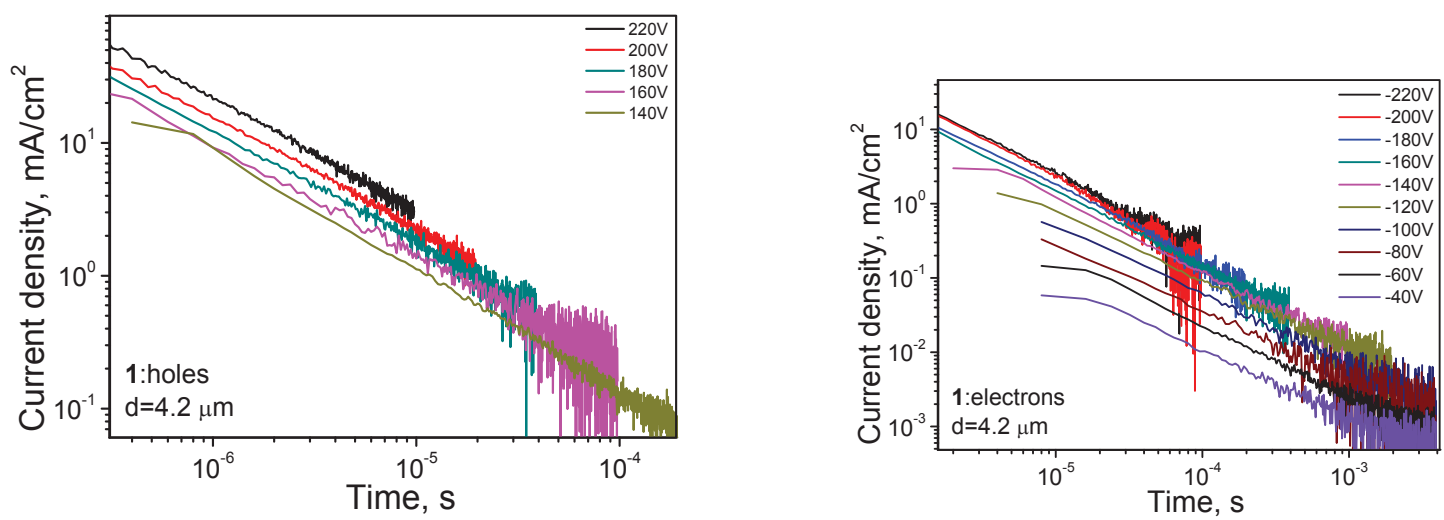

Figure S22. TOF pulses, obtained by applying positive or negative external electric voltages to the samples, for holes and electrons in the layers of studied compounds.
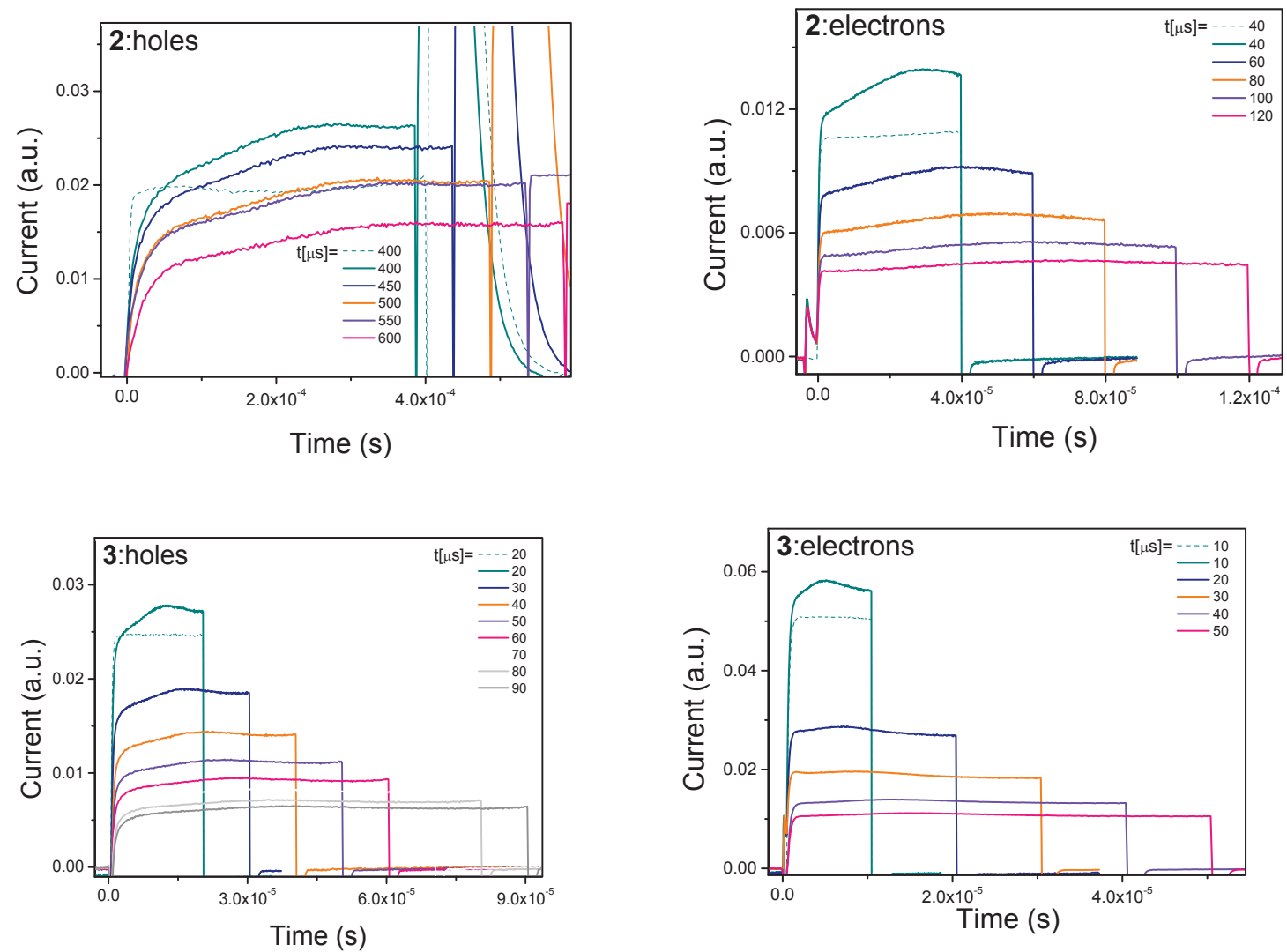

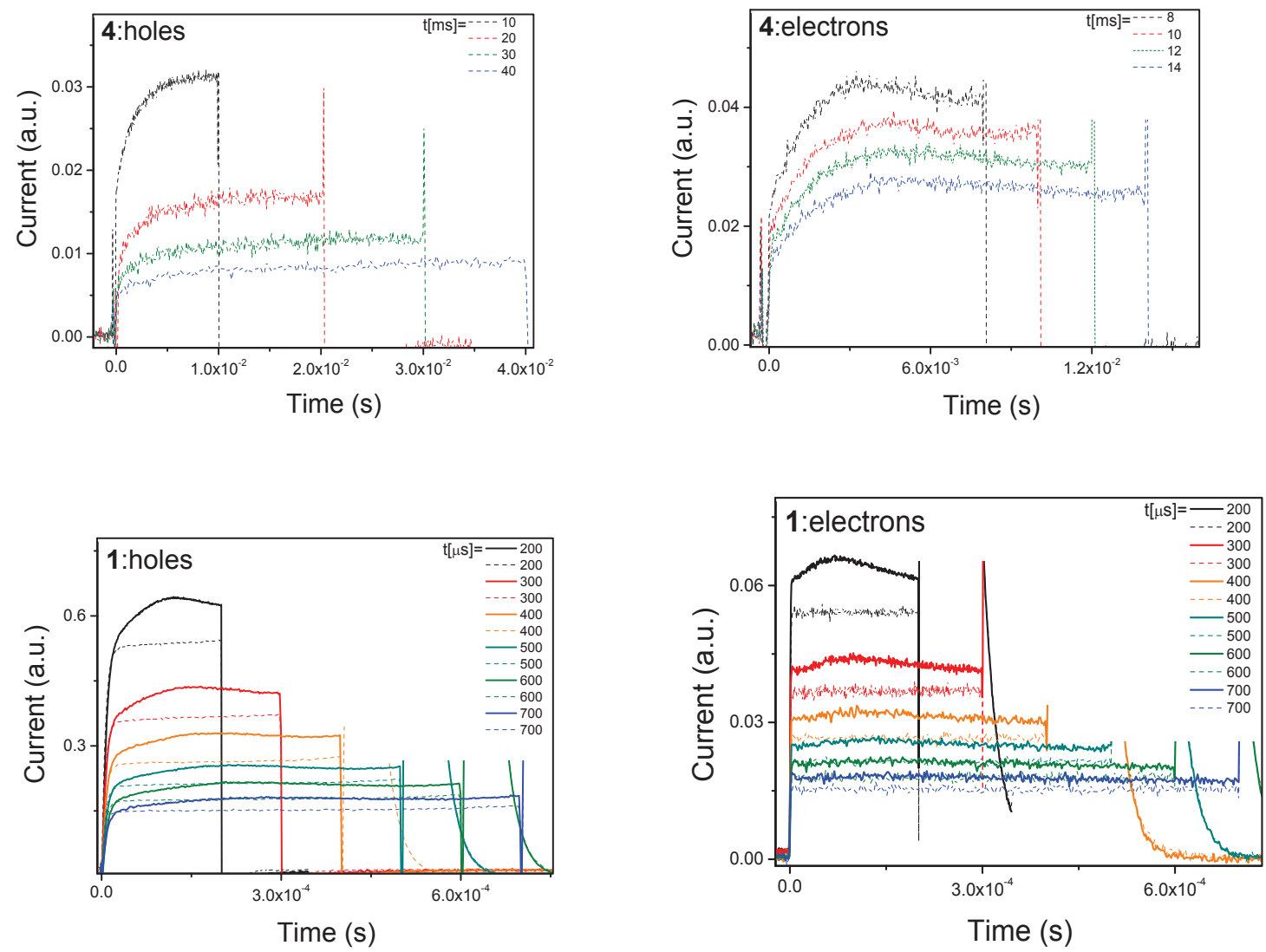

Figure S23. Dark-CELIV (dash lines) and photo-CELIV (straight lines) signals for holes and electrons in the layers of studied compounds.

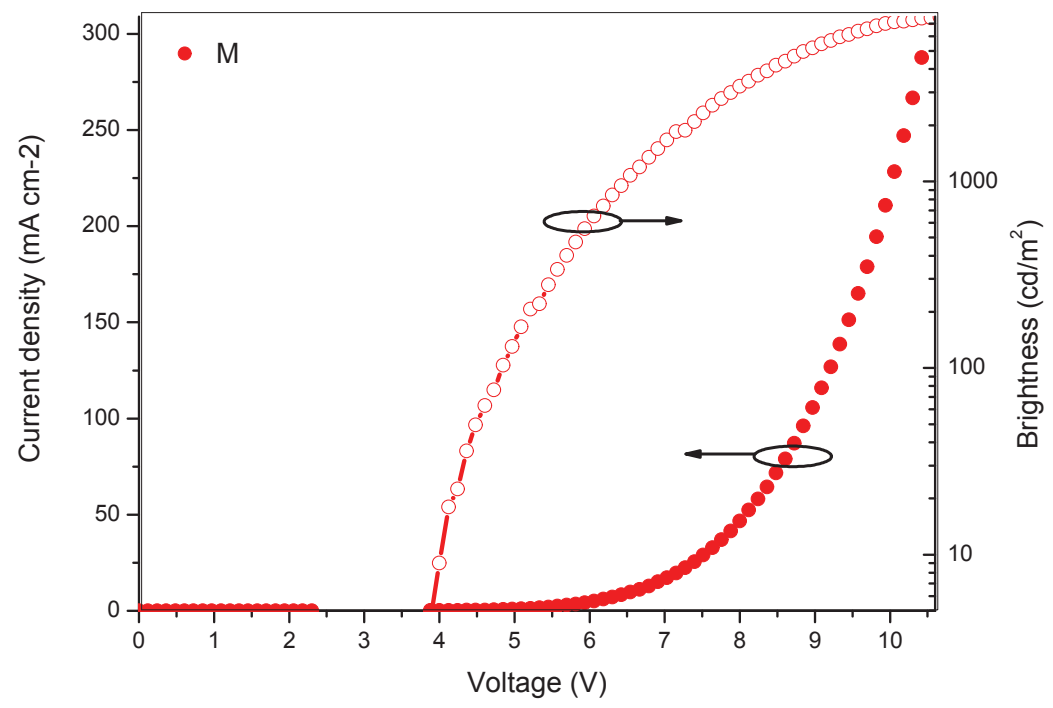

Figure S24. Current density and brightness of the M OLED. 


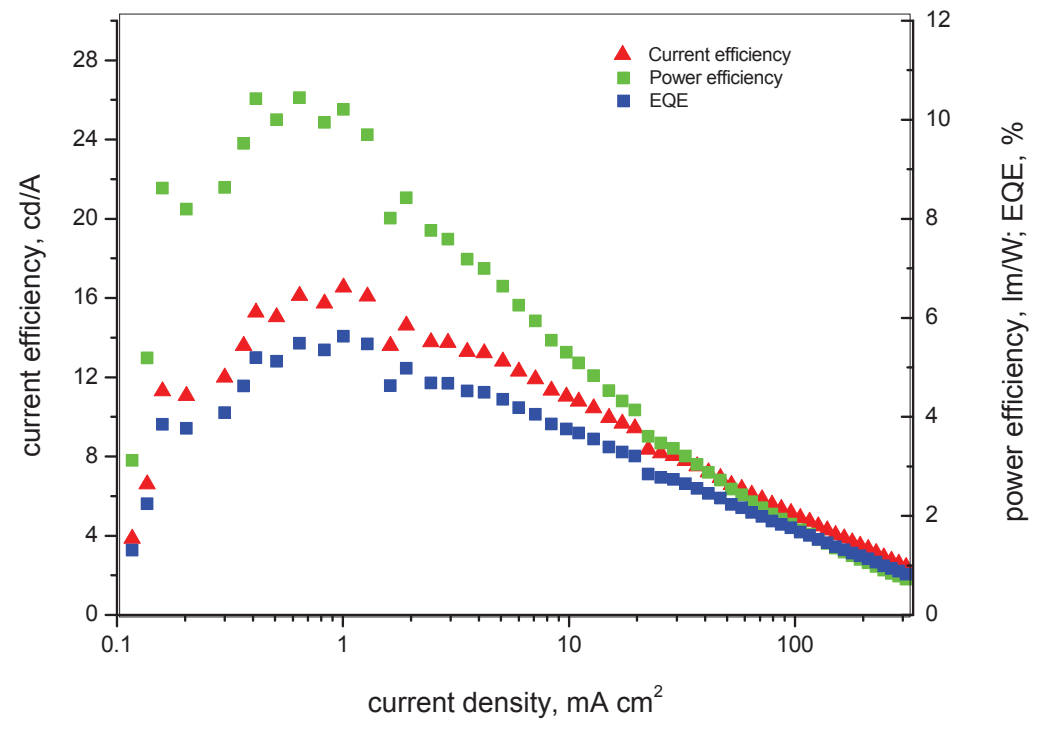

Figure S25. Current, power and external quantum efficiency of the M OLED.

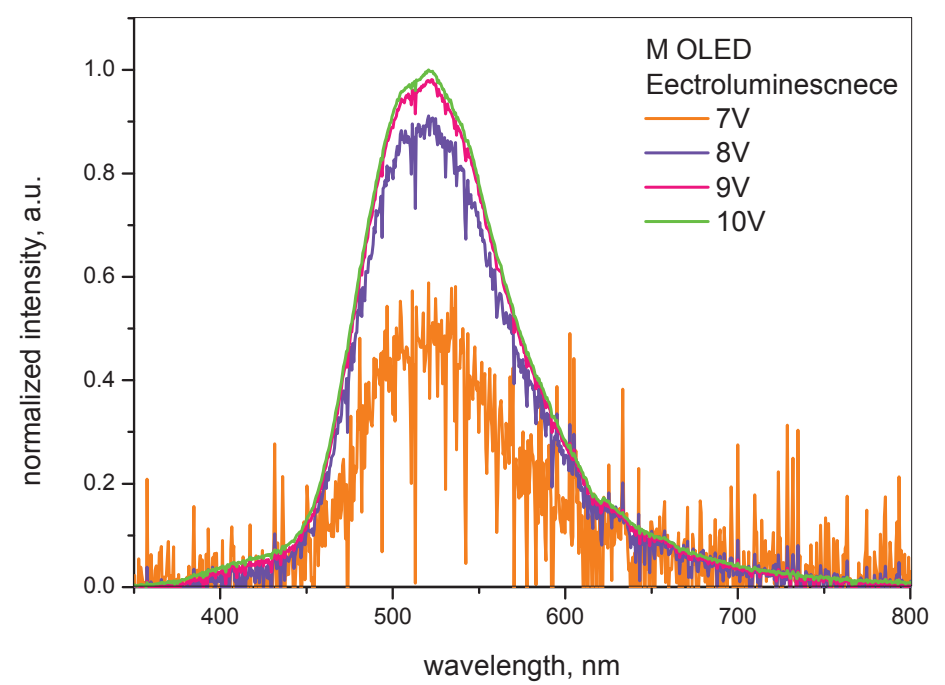

Figure S26. Electroluminescence spectra of the M OLED.

\section{NMR spectra}



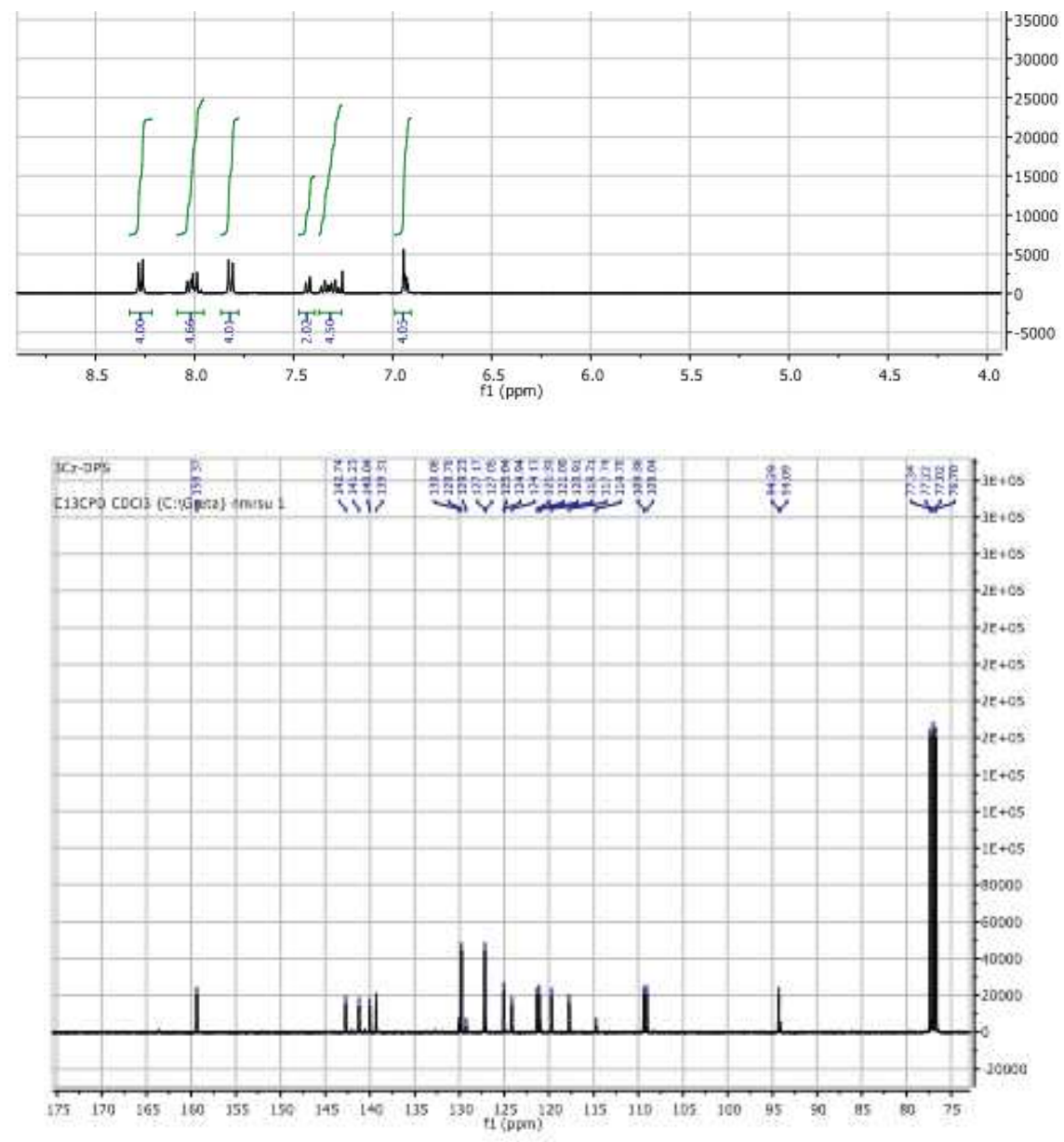

Figure. S27. ${ }^{1} \mathrm{HNMR}$ and ${ }^{13} \mathrm{CNMR}$ spectra of 1 recorded in $\mathrm{CDCl}_{3}$.

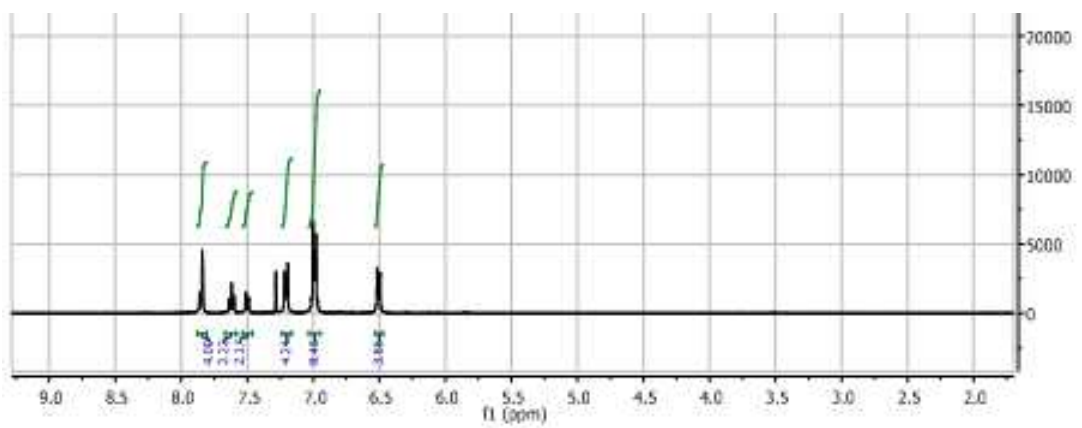




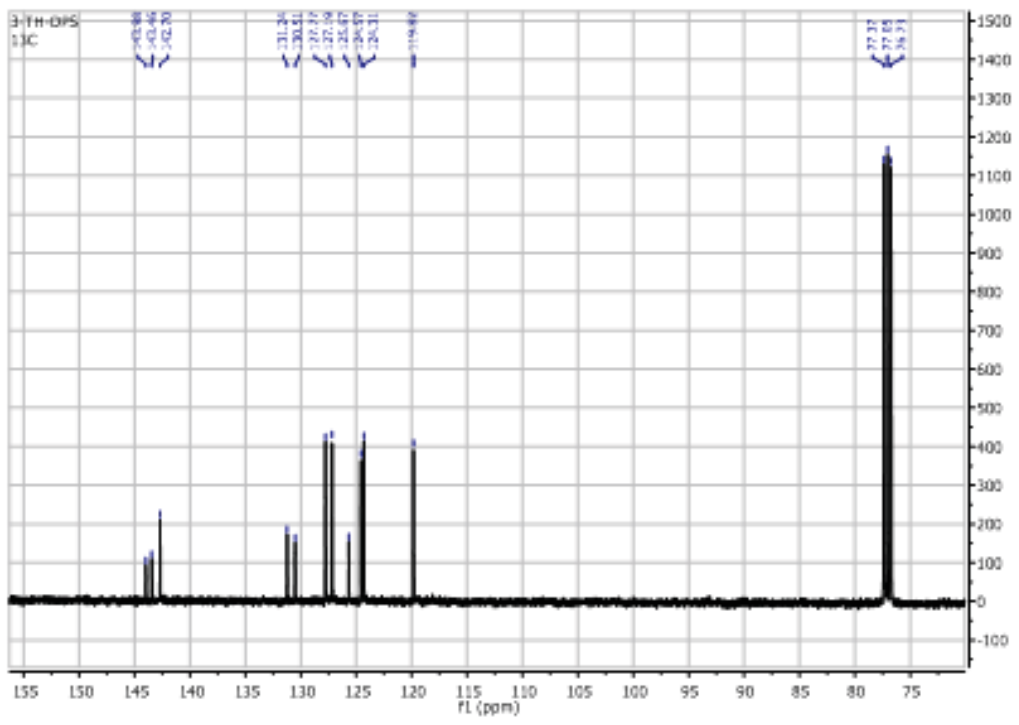

Figure S28. ${ }^{1} \mathrm{HNMR}$ and ${ }^{13} \mathrm{CNMR}$ spectra of 2 recorded in $\mathrm{CDCl}_{3}$.
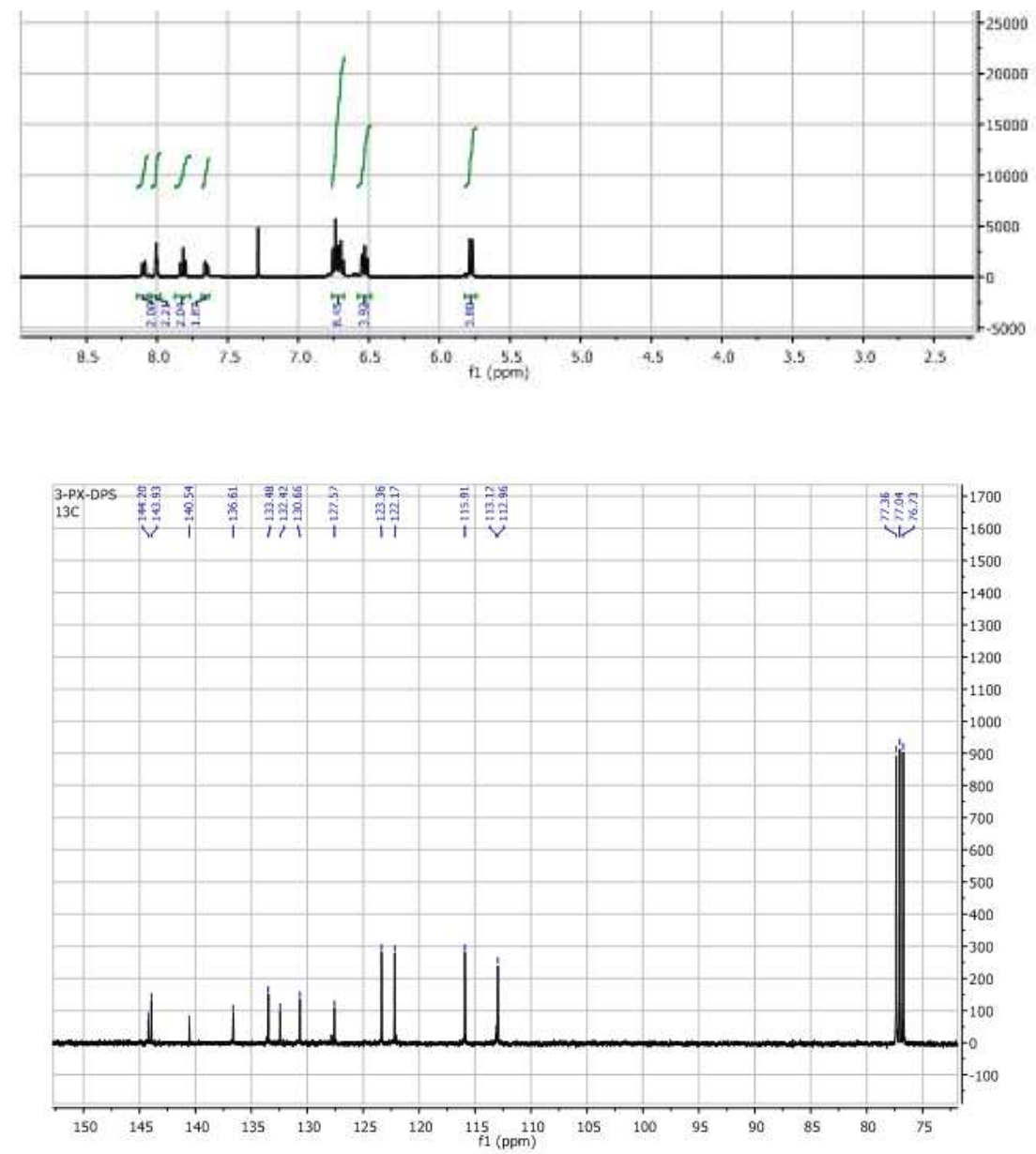

Figure S29. ${ }^{1} \mathrm{HNMR}$ and ${ }^{13} \mathrm{CNMR}$ spectra of 3 recorded in $\mathrm{CDCl}_{3}$. 

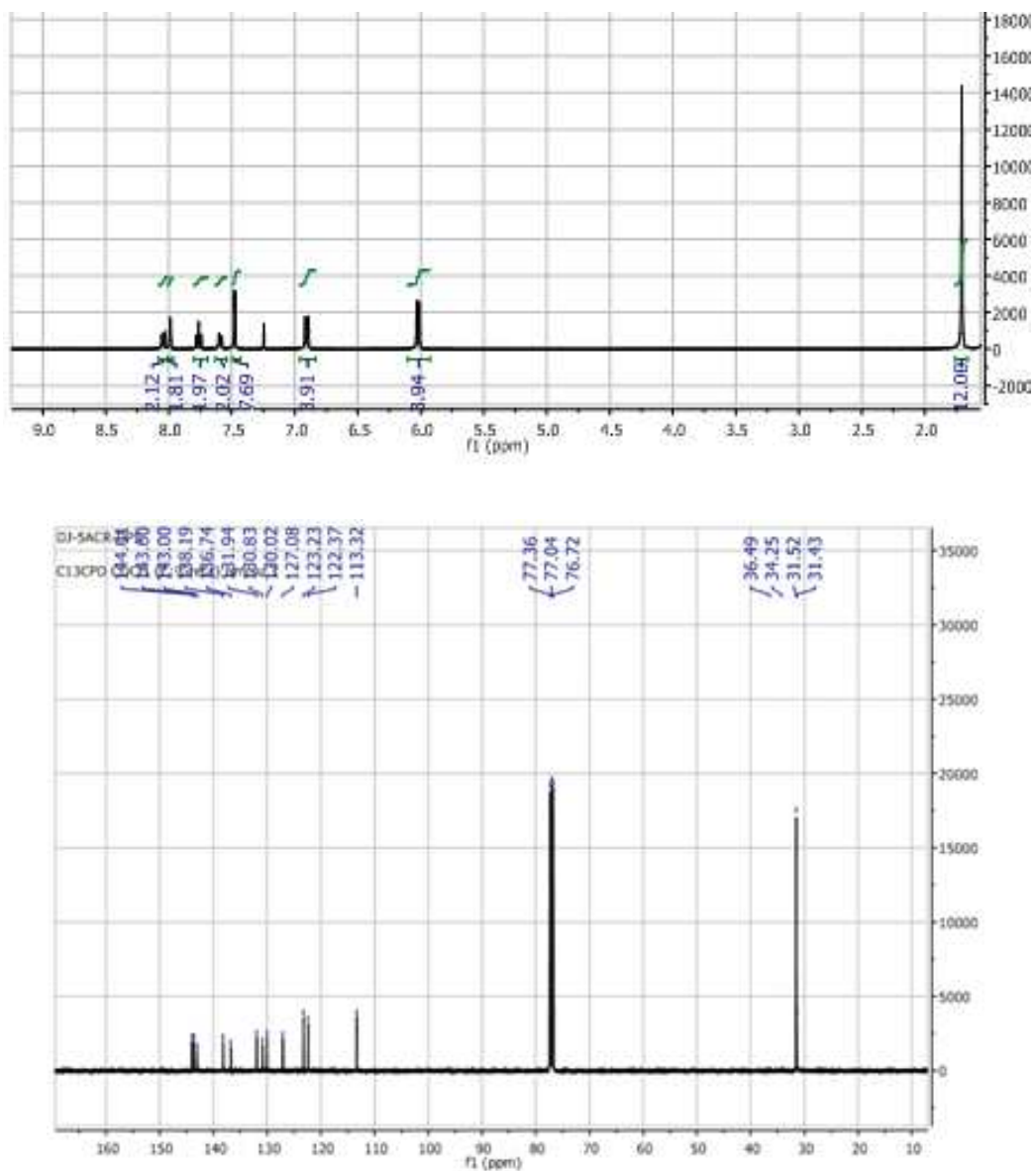

Figure S30. ${ }^{1} \mathrm{HNMR}$ and ${ }^{13} \mathrm{CNMR}$ spectra of 4 recorded in $\mathrm{CDCl}_{3}$.

This paper is elaborated at the Centre of Excellence in Advanced $M$ aterial Research and Technology Transfer of the Institute of Solid State Physics, University of Latvia executing project CAM ART2 ${ }^{2}$ that has received funding from the Horizon 2020 Framework Programme H2020-WIDESPREAD-01-2016-2017-TeamingPhase2 under grant agreement No. 739508 